\title{
Nearly neighbourly families of standard boxes
}

\author{
Jacek Bojarski Andrzej Kisielewicz Krzysztof Przesławski \\ The Faculty of Mathematics, Computer Science and Econometrics \\ University of Zielona Gora \\ Zielona Góra, Poland \\ \{j.bojarski, a.kisielewicz, k.przeslawski\}@wmie.uz.zgora.pl
}

Submitted: Jan 21, 2017; Accepted: Nov 14, 2019; Published: Dec 6, 2019

(C) The authors. Released under the CC BY-ND license (International 4.0).

\begin{abstract}
It is shown that each nearly neighbourly family of standard boxes in $\mathbb{R}^{3}$ has at most 12 elements. A combinatorial classification of all such families that have exactly 12 elements is given. All the families satisfying an extra property called incompressibility are described. Compressible families are discussed briefly.
\end{abstract}

Mathematics Subject Classifications: 52B10, 05C69

\section{Introduction}

A standard $n$-box or an $n$-interval is the Cartesian product of $n$ ordinary closed intervals of positive length. Two $n$-intervals $I=I_{1} \times \cdots \times I_{n}$ and $J=J_{1} \times \cdots \times J_{n}$ are adjacent if there is $i \in[n]$ such that $I_{i}$ and $J_{i}$ have exactly one point in common. Both the family and the infinite graph of all $n$-intervals with the adjacency just defined are denoted by $\mathscr{I}^{n}$. This convention extends to any subfamily $\mathscr{G}$ of $\mathscr{I}^{n}$ : The same symbol $\mathscr{G}$ is for the graph with adjacency inherited from $\mathscr{I}^{n}$. A subfamily $\mathscr{G} \subset \mathscr{I}^{n}$ is nearly neighbourly if it is a clique in $\mathscr{I}^{n}$.

The main purpose of the present investigation is to demonstrate that the maximum cardinality of a nearly neighbourly family in $\mathscr{I}^{3}$ is 12 (Theorem 16), and to give full combinatorial and geometric descriptions of all such families. It should be pointed out that the case of the so-called compressible cliques (see Section 6) is addressed rather superficially. Only results without proofs are presented. A more detailed analysis will possibly be published elsewhere.

The notion of a nearly neighbourly family of intervals is a specialization of a more general concept promoted by Zaks [16]: A family $\mathscr{P}$ of $n$-dimensional convex polytopes in $\mathbb{R}^{n}$ is said to be nearly neighbourly, if for every two polytopes $P, Q$ belonging to $\mathscr{P}$ there is a hyperplane separating them that contains a facet of $P$ and a facet of $Q$. In fact, researchers have paid more attention to neighbourly families. Let us remind that $\mathscr{P}$ is 
neighbourly if the intersection of any two members of $\mathscr{P}$ is of dimension $n-1$. In general, such a $\mathscr{P}$ can be of arbitrary finite cardinality unless $n \leqslant 2$; in addition, one may even assume that the members of $\mathscr{P}$ are affinely equivalent [11] or congruent [5]. However, if a nearly neighbourly family $\mathscr{P}$ consists of polytopes having their number of facets bounded from the above by $m$, then, as mentioned in [17],

$$
|\mathscr{P}| \leqslant 2^{m}
$$

The proof is based on an idea of Perles [13]. (A similar method has been employed by other researches even earlier; see [6] for further details). It is conjectured that if $\mathscr{P}$ consists of $n$-dimensional simplices, then the above estimate can be improved; that is, $|\mathscr{P}| \leqslant 2^{n}$. The conjecture is open for all $n \geqslant 3$. (The best known estimate is $2^{n+1}-2$; see $[7,18]$, where the case $n=3$ is considered, and [9] for general $n$ ). It is even open for neighbourly families of simplices in dimension 4. As it concerns tetrahedra, it was Bagemihl [3] who raised the question. He constructed a neighbourly family of 8 tetrahedra, and speculated that it is a family of maximum cardinality. Subsequently, Baston [4] proved that a neighbourly family of tetrahedra has at most 9 elements. And finally Zaks [18] was able to show that it has at most 8 elements as expected. His work depends heavily on Baston's research and the Graham-Pollak theorem [8] on minimal biclique partitions of complete graphs. It was also Zaks [19] who constructed a neighbourly family of $n$-dimensional simplices consisting of $2^{n}$ members for $n>3$.

We know just a couple of papers devoted to (nearly) neighbourly families of standard boxes. Zaks [20] proved that the maximum cardinality of a neighbourly family of $n$ intervals is $n+1$. Again, the proof depends on the Graham-Pollak theorem. In [2], $\mathrm{N}$. Alon studied $k$-neighbouring families of $n$-intervals. Let us remind that $\mathscr{P}$ is such a family if for every two members $P$ and $Q$ of $\mathscr{P}$ one has $n-k \leqslant \operatorname{dim} P \cap Q \leqslant n-1$. He gave estimates from below and above for the maximum cardinality of a $k$ neighbourly family of $n$-intervals. There are two works $[14,15]$ by J. D. Simon on (nearly) neighbourly families of quadrilaterals. Some of her results will be discussed in Section 4 .

We begin with showing that for families of $n$-intervals Perles' estimate (1) can be improved by a constant factor (Proposition 10, Remark 3). In this context, we introduce a bulk of notions instrumental for further presentation.

\section{Preliminaries}

Let $X$ be a non-empty set. We denote by $\mathbb{N} X$ the abelian semigroup of all finite formal sums of elements of $X$. (The elements of $\mathbb{N} X$ will also be called combinations). Every combination $\gamma \in \mathbb{N} X$ is uniquely determined by a function $k: X \rightarrow \mathbb{N}$ with support $\left\{x: k_{x}>0\right\}$ of finite cardinality. We shall use the following notation related to $\gamma$ :

$$
\gamma=\sum_{x \in X} k_{x} x=\sum k_{x} x, \quad|\gamma|=\sum k_{x}, \quad \operatorname{supp} \gamma=\left\{x: k_{x}>0\right\} .
$$

On several occasions we will use a naturally defined partial order on $\mathbb{N} X$ : For $\delta=\sum l_{x} x$ and $\gamma=\sum k_{x} x$, we write $\delta \leqslant \gamma$ if and only if $l_{x} \leqslant k_{x}$, for every $x \in X$. If $\delta \leqslant \gamma$, then we 
say that $\delta$ is a subcombination of $\gamma$. If $l_{x} \in\left\{k_{x}, 0\right\}$, for every $x \in X$, then $\delta$ is an induced subcombination of $\gamma$. Suppose $G$ is a graph whose set of vertices $V(G)$ equals $X$. Then each $\gamma$ determines in a natural way the graph $G_{\gamma}$ :

$$
\begin{aligned}
& V\left(G_{\gamma}\right)=\bigcup_{x: k_{x}>0}\left\{(x, 1), \ldots,\left(x, k_{x}\right)\right\} \\
& E\left(G_{\gamma}\right)=\left\{\{(x, i),(y, j)\}:\{x, y\} \in E(G), 1 \leqslant i \leqslant k_{x}, 1 \leqslant j \leqslant k_{y}\right\} .
\end{aligned}
$$

In what follows, we shall often appeal to $\gamma$ itself as to $G_{\gamma}$. Consequently, we shall write $V(\gamma), E(\gamma)$ rather than $V\left(G_{\gamma}\right), E\left(G_{\gamma}\right)$.

Proposition 1. Let $G$ be a graph and let $\gamma=\sum k_{x} x$ be an element of $\mathbb{N} V(G)$. Then

$$
|V(\gamma)|=\sum k_{x}, \quad|E(\gamma)|=\sum_{\{x, y\} \in E(G)} k_{x} k_{y} .
$$

The clique number $\omega(G)$ of a graph $G$ is the cardinality of a maximum clique contained in $V(G)$. If $\gamma \in \mathbb{N} V(G)$, Then $\omega(\gamma)=\omega\left(G_{\gamma}\right)$ is equal to $\omega(G[\operatorname{supp} \gamma])$, the clique number of the subgraph of $G$ induced by supp $\gamma$. The independence number $\alpha(G)$ of $G$ is the clique number of the complement graph of $G$.

Suppose that two non-empty sets $X$ and $Y$, and a mapping $f: X \rightarrow Y$ are given. Then $f$ induces the mapping $f_{*}: \mathbb{N} X \rightarrow \mathbb{N} Y$

$$
f_{*}(\gamma)=\sum k_{x} f(x)
$$

Proposition 2. Given two graphs $G$ and $H$, and a homomorphism of graphs $f: V(G) \rightarrow$ $V(H)$. Then for every $\gamma \in \mathbb{N} V(G)$, the graph $f_{*}(\gamma)$ is a homomorphic image of $\gamma$. In particular, $\alpha\left(f_{*}(\gamma)\right) \leqslant \alpha(\gamma)$.

Our next proposition is rather obvious.

Proposition 3. Let $G_{1}$ and $G_{2}$ be two graphs and let $G=G_{1} * G_{2}$ be their disjunctive product; that is, $V(G)=V\left(G_{1}\right) \times V\left(G_{2}\right)$ and $\left\{\left(u_{1}, u_{2}\right),\left(v_{1}, v_{2}\right)\right\} \in E(G)$ if and only if $\left\{u_{1}, v_{1}\right\} \in E\left(G_{1}\right)$ or $\left\{u_{2}, v_{2}\right\} \in E\left(G_{2}\right)$. Let $C$ be a finite clique in $G$ and $\gamma_{i}=\sum_{x \in C} x_{i}$, $i=1,2$. Then

$$
\alpha\left(\gamma_{1}\right) \leqslant \omega\left(\gamma_{2}\right)
$$

Proof. Let $D \subseteq C$ be a set of maximum cardinality such that $\sum_{x \in D} x_{1}$ is independent. Then $\sum_{x \in D} x_{2}$ is a clique and $|D|=\alpha\left(\gamma_{1}\right)$. Therefore,

$$
\alpha\left(\gamma_{1}\right)=\left|\sum_{x \in D} x_{2}\right| \leqslant \omega\left(\gamma_{2}\right) .
$$

Proposition 4. Let $K=\left\{i_{1}<i_{2}<\ldots<i_{k}\right\}$ be a proper subset of $[n]$. Let $K^{\mathrm{c}}$ be its complement. For $I \in \mathscr{I}^{n}$, let $I_{K}=I_{i_{1}} \times \cdots \times I_{i_{k}}$. Then the mapping $I \mapsto\left(I_{K}, I_{K^{c}}\right)$ defines an isomorphism between graphs $\mathscr{I}^{n}$ and $\mathscr{I}|K| * \mathscr{I}^{\left|K^{c}\right|}$. 
By Propositions 3 and 4, we have

Corollary 5. Let $K$ be a proper subset of $[n]$. Let $\mathscr{C}$ be a clique in $\mathscr{I}^{n}$. Let $\gamma_{L}=$ $\sum_{I \in \mathscr{C}} I_{L}$, where $L=K, K^{\mathrm{c}}$. Then

$$
\alpha\left(\gamma_{K}\right) \leqslant \omega\left(\gamma_{K^{c}}\right) .
$$

In what follows, the notation of Corollary 5 is employed in a more general setting: Let $\gamma=\sum_{I} k_{I} I \in \mathbb{N} \mathscr{I}^{n}$, and $K$ be a proper subset of $[n]$; then $\gamma_{K}=\sum_{I} k_{I} I_{K}$. If $K=\{i\}$, then we shall often write $\gamma_{i}$ rather than $\gamma_{\{i\}}$.

\section{Incompressibility. An upper bound for the cardinality of a maximum clique in $\mathscr{I}^{n}$.}

For $s \in \mathbb{N}$, let $\mathscr{I}(s)=\{[0,1],[s, s+1]\} \cup\{[i, j]: 1 \leqslant i<j \leqslant s\}$. Let $\mathscr{G}$ be a finite subfamily of $\mathscr{I}^{1}$. There is clearly a homomorphism of graphs $f: \mathscr{G} \rightarrow \mathscr{I}(s)$ for some $s$ (for example, but not necessarily, one obtained by a homeomorphism of $\mathbb{R}$ that sends all endpoints of intervals in $\mathscr{G}$ to positive integers). We shall be concerned with properties of the family $f(\mathscr{G})$ for minimal $s$.

Proposition 6. Let a finite nonempty family $\mathscr{G} \subseteq \mathscr{I}^{1}$ be given. Let $s=s(\mathscr{G})$ be the minimum number for which there is a homomorphism of graphs $f: \mathscr{G} \rightarrow \mathscr{I}(s)$. Then

(1) $\{[i, i+1]: 0 \leqslant i \leqslant s\} \subseteq f(\mathscr{G})$;

(2) $\alpha(\mathscr{G}) \geqslant\left\lfloor\frac{s}{2}\right\rfloor+1$;

(3) $\{[1,3],[s-2, s]\} \subseteq f(\mathscr{G})$, whenever $s \geqslant 3$.

Proof. There is nothing to prove if $s=0$. The case $s=1$ is rather obvious. Moreover, $s \neq 2$ as the mapping [2,3] $\mapsto[0,1],[i, i+1] \mapsto[i, i+1]$ for $i=0,1$ is a homomorphism of $\mathscr{I}(2)$ onto $\mathscr{I}(1)$. Therefore we may assume $s \geqslant 3$. Suppose $[0,1] \notin f(\mathscr{G})$. For every $I=[a, b]$ belonging to $f(\mathscr{G})$, let as set

$$
I^{\prime}= \begin{cases}{[1, b-1]} & \text { if } a=0 \text { or } a=1, b>2, \\ {[a-1, b-1]} & \text { if } a=1, b=2 \text { or } a>1 .\end{cases}
$$

The mapping $g$ defined by $I^{\prime}=g(I)$ is a homomorphism of $f(\mathscr{G})$ into $\mathscr{I}(s-1)$. Therefore, the composite $g \circ f$ maps $\mathscr{G}$ into $\mathscr{I}(s-1)$ contrary to the definition of $s$. Since the mapping $[a, b] \stackrel{h}{\mapsto}[s+1-b, s+1-a]$ is an automorphism of $\mathscr{I}(s)$ which sends $[s, s+1]$ on $[0,1]$, the interval $[s, s+1]$ has to belong to $f(\mathscr{G})$ also.

Suppose now that $[i, i+1] \notin f(\mathscr{G})$, where $i \in[1, s-1]$. Then define the correspondence $I=[a, b] \stackrel{g}{\mapsto} I^{\prime}$ as follows

$$
I^{\prime}= \begin{cases}{[a, b]} & \text { if } b \leqslant i \\ {[a, b-1]} & \text { if } a \leqslant i, b \geqslant i+1 \\ {[a-1, b-1]} & \text { if } a \geqslant i+1\end{cases}
$$


Again, the composite $g \circ f$ is a homomorphism of $\mathscr{G}$ into $\mathscr{I}(s-1)$ contradicting the minimality of $s$.

In order to prove (2), it suffices to observe that by (1), the independent set of intervals $\left\{[2 i, 2 i+1]: 0 \leqslant i \leqslant\left\lfloor\frac{s}{2}\right\rfloor\right\}$ is contained in $f(\mathscr{G})$, and that $\alpha(f(\mathscr{G})) \leqslant \alpha(\mathscr{G})$.

As it concerns the intervals $[1,3]$ and $[s-2, s]$, the automorphism $h$ transposes them. Moreover, there is an automorphism (see Appendix A) which transposes $[1,3]$ with $[2,3]$. Therefore, both $[1,3]$ and $[s-2, s]$ have to belong to $f(\mathscr{G})$.

REMARK 1. Suppose $\mathscr{G} \subset \mathscr{I}^{1}$ consists of intervals of odd length with integer endpoints. Then the mapping $[a, b] \mapsto[a \bmod 2,1+(a \bmod 2)]$ is a graph homomorphism from $\mathscr{G}$ to $\mathscr{I}(1)$. If there are two intervals in $\mathscr{G}$ with right endpoints of different parity, then $s(\mathscr{G})=2$ and consequently $\mathscr{G}$ is bipartite. If all intervals in $\mathscr{G}$ have their right endpoints of equal parity, then $s(\mathscr{G})=0$ and $\mathscr{G}$ has no edges.

A subgraph $\mathscr{G} \subseteq \mathscr{I}^{1}$ is incompressible if there is $s$ such that $\mathscr{G} \subseteq \mathscr{I}(s)$ and there is no a homomorphism $f: \mathscr{G} \rightarrow \mathscr{I}\left(s^{\prime}\right)$, where $s^{\prime}<s$. Otherwise, $\mathscr{G}$ is called compressible. A combination $\gamma \in \mathbb{N} \mathscr{I}(s)$ is incompressible (compressible) if supp $\gamma$ is an incompressible (compressible) subgraph of $\mathscr{I}(s)$. As an immediate consequence of the preceding proposition we have

Proposition 7. If $\gamma \in \mathbb{N} \mathscr{I}(s) \backslash\{0\}$ is incompressible then

(1) $s \leqslant 2 \alpha(\gamma)-1$

(2) $\mathscr{S}(s)=\{[i, i+1]: 0 \leqslant i \leqslant s\} \cup\{[1,3],[s-2, s]\}$ is contained in supp $\gamma$, whenever $s \geqslant 3$.

Lemma 8. Let $\gamma \in \mathbb{N} \mathscr{I}^{1}$ be given. If $\alpha(\gamma)>1$, then

$$
|\gamma| \leqslant 4 \alpha(\gamma)-3
$$

Proof. We may assume that $\operatorname{supp} \gamma \subseteq \mathscr{I}(s)$ for some $s$. If $\gamma$ is compressible, then let us choose a new $s$ and a homomorphism $f: \operatorname{supp} \gamma \rightarrow \mathscr{I}(s)$ so that $\gamma^{\prime}=f_{*}(\gamma)$ is incompressible. If $s=0$ or $s=1$, then since $\alpha(\gamma)>1$, we obtain

$$
|\gamma|=\left|\gamma^{\prime}\right| \leqslant 2 \alpha\left(\gamma^{\prime}\right) \leqslant 2 \alpha(\gamma) \leqslant 4 \alpha(\gamma)-3
$$

As $\mathscr{I}(2)$ is compressible, we may assume that $s>2$. Then, by the preceding lemma, $[0,1]$ and $[2,3]$ are elements of supp $\gamma^{\prime}$ and consequently $\alpha\left(\gamma^{\prime}\right)>1$. Henceforth, we may assume that $\gamma \in \mathbb{N} \mathscr{I}(s)$ is incompressible and $s>2$. Let us set $B=\{0,1\}^{s}$ and

$$
B(I)= \begin{cases}\{1\} \times\{0,1\}^{s-1}, & \text { if } I=[0,1] \\ \{0,1\}^{i-1} \times\{0\} \times\{0,1\}^{j-i-1} \times\{1\} \times\{0,1\}^{s-j}, & \text { if } I=[i, j], 1 \leqslant i<j \leqslant s \\ \{0,1\}^{s-1} \times\{0\}, & \text { if } I=[s, s+1]\end{cases}
$$

Let $\gamma^{\sharp}: B \rightarrow \mathbb{R}$ be associated with $\gamma=\sum_{I \in \mathscr{I}(s)} k_{I} I$ by the formula

$$
\gamma^{\sharp}(x)=\sum_{I \in \mathscr{I}(s)} k_{I} \mathbf{1}_{B(I)}(x)
$$


Observe that $\gamma^{\sharp}$ is bounded from the above by $\alpha(\gamma) \mathbf{1}_{B}$. Moreover, these two functions do not coincide, as if $x_{0}=(0, \ldots, 0)$, then, bearing in mind that Proposition 6 guarantees $k_{[0,1]}>0$, we have

$$
\gamma^{\sharp}\left(x_{0}\right)=k_{[s, s+1]}<k_{[0,1]}+k_{[s, s+1]} \leqslant \alpha(\gamma) .
$$

Therefore, summing up these functions with respect to $x$ yields

$$
\alpha(\gamma)|B|>\sum_{I} k_{I}|B(I)|
$$

If $I=[0,1]$ or $I=[s, s+1]$, then $|B(I)|=\frac{1}{2}|B|$; if not, $|B(I)|=\frac{1}{4}|B|$. By Proposition 6, $k_{[0,1]}$ and $k_{[s, s+1]}$ are both greater or equal to 1 , thus

$$
\alpha(\gamma)>\frac{k_{[0,1]}+k_{[s, s+1]}}{4}+\frac{1}{4} \sum_{I \in \mathscr{I}(s)} k_{I} \geqslant \frac{1}{2}+\frac{|\gamma|}{4} .
$$

We denote by $b_{m}$ the maximum cardinality of a clique in $\mathscr{I}^{m}$.

Proposition 9. Let $K$ be a proper subset of $[n]$. Let $\mathscr{C}$ be a clique in $\mathscr{I}^{n}$. Let $\gamma_{K}=$ $\sum_{I \in \mathscr{C}} I_{K}$. Then

$$
\alpha\left(\gamma_{K}\right) \leqslant b_{n-|K|}
$$

Proof. By Corollary 5 and the fact that $\operatorname{supp}\left(\gamma_{K^{c}}\right) \subset \mathscr{I}^{n-|K|}$, one has

$$
\alpha\left(\gamma_{K}\right) \leqslant \omega\left(\gamma_{K^{c}}\right)=\omega\left(\operatorname{supp}\left(\gamma_{K^{c}}\right)\right) \leqslant \omega\left(\mathscr{I}^{n-|K|}\right)=b_{n-|K|} .
$$

Proposition 10. For every $n \geqslant 2$

$$
b_{n} \leqslant 4 b_{n-1}-3
$$

Moreover, $b_{2}=5$.

Proof. Suppose $\mathscr{C}$ to be a finite clique in $\mathscr{I}^{n}$. Let $\gamma_{1}=\sum_{I \in \mathscr{C}} I_{1}$. By Lemma 8 and Proposition 9, one gets

$$
|\mathscr{C}|=\left|\gamma_{1}\right| \leqslant 4 \alpha\left(\gamma_{1}\right)-3 \leqslant 4 b_{n-1}-3
$$

whenever $\alpha\left(\gamma_{1}\right)>1$. If $\alpha\left(\gamma_{1}\right)=1$, then $\left|\gamma_{1}\right| \leqslant 2$ and the inequality $|\mathscr{C}| \leqslant 4 b_{n-1}-3$ is still valid.

To prove the second part, observe that since $b_{1}=2$, it follows by the first part that $b_{2} \leqslant 5$. Any of the configurations depicted in Figure 2 shows that $b_{2}$ is at least 5 .

REMark 2. The fact that $b_{2}=5$ is due to Simon [14, Theorem 5.7]. Her proof seems to be different. It depends on the analysis of the so-called Baston matrix of a maximum family of 2-intervals.

Corollary 11 . Let $k \geqslant 1$ and $n \geqslant k$. Then

$$
b_{n} \leqslant \frac{b_{k}-1}{4^{k}} 4^{n}+1 \text {. }
$$


REMARK 3. As we have announced, we shall demonstrate later on that $b_{3}=12$. Therefore, for every $n \geqslant 3$

$$
b_{n} \leqslant \frac{11}{64} 4^{n}+1
$$

Remark 4. If $\mathscr{C}$ is a clique in $\mathscr{I}^{k}$ and $\mathscr{D}$ is a clique in $\mathscr{I}^{l}$, then $\mathscr{E}=\{I \times J: I \in \mathscr{C}, J \in \mathscr{D}\}$ is a clique in $\mathscr{I}^{k+l}$. Therefore, we have

$$
b_{k} b_{l} \leqslant b_{k+l}
$$

In particular, for every $n>1$

$$
b_{n} \geqslant 2 b_{n-1},
$$

as $b_{1}=2$.

\section{Maximum cliques in $\mathscr{I}^{2}$}

We are going to describe all cliques of maximal cardinality in $\mathscr{I}^{2}$. Let $\mathscr{C}$ be such a clique. By Proposition 10, $|\mathscr{C}|=5$. Let $E_{i}=\left\{\{I, J\} \in E(\mathscr{C}): I_{i}\right.$ and $J_{i}$ are adjacent in $\left.\mathscr{I}^{1}\right\}$, $i=1,2$. Let the graphs $G_{i}$ be defined by $V\left(G_{i}\right)=\mathscr{C}, E\left(G_{i}\right)=E_{i}$. Let $\gamma_{i}=\sum_{I \in \mathscr{C}} I_{i}$. It is clear that $G_{i}$ is isomorphic to the graph associated with $\gamma_{i}$. Since $\mathscr{I}^{1}$ does not contain triangles, $\gamma_{i}$ and consequently $G_{i}$ cannot contain triangles either. By the definition of adjacency in $\mathscr{I}^{2}$, the sets $E_{1}, E_{2}$ cover $E(\mathscr{C})$. These facts imply that the graphs $G_{i}$ have no vertices of degree higher than two and $\left|E_{i}\right| \leqslant 5$. As $\mathscr{C}$ is a 5-clique, its set of edges $E(\mathscr{C})$ has exactly ten elements. Therefore, $\left\{E_{1}, E_{2}\right\}$ is a partition of $E(\mathscr{C})$ and $\left|E_{i}\right|=5$ for $i=1,2$. It is rather clear now that $G_{1}, G_{2}$ gives us a decomposition of $\mathscr{C}$ into two 5 -cycles.

Given a strictly increasing sequence of numbers $a_{0}, \ldots, a_{4}$. Then

$$
\Gamma=\left\{\left[a_{k}, a_{k+1}\right]: k=0, \ldots, 3\right\} \cup\left\{\left[a_{1}, a_{3}\right]\right\} .
$$

is a 5 -cycle in $\mathscr{I}^{1}$. It is easy to verify, that all 5 -cycles in $\mathscr{I}^{1}$ are of this form. Let $\gamma \in \mathbb{N} \mathscr{I}^{1}$, be a 5 -cycle. It is equally easy to observe that there is a unique 5 -cycle $\Gamma \in \mathscr{I}^{1}$ such that $\gamma=\sum_{I \in \Gamma} I$. Henceforth, we have just reduced the problem of describing all cliques of maximal cardinality in $\mathscr{I}^{2}$ to the following construction problem:

Given two 5 -cycles $\Gamma_{i}, i=1,2$ in $\mathscr{I}^{1}$. Find all possible 5 -cliques $\mathscr{C} \subset \mathscr{I}^{2}$ such that

$$
\mathscr{C}_{i}:=\left\{I_{i}: I \in \mathscr{C}\right\}=\Gamma_{i} .
$$

Let $a_{i, 0}, \ldots, a_{i, 4}$, be the sequence defining the 5 -cycle $\Gamma_{i}$ for $i=1,2$. We can label each interval belonging to $\Gamma_{1}$ as follows:

$$
\left[a_{1, k}, a_{1, k+1}\right] \mapsto k+1 \text { for } k=0, \ldots, 3 \text { and }\left[a_{1,1}, a_{1,3}\right] \mapsto 5 \text {. }
$$

Since $I \mapsto I_{1}$ is a one-to-one mapping from $\mathscr{C}$ onto $\Gamma_{i}$ for every $\mathscr{C}$ to be constructed, it determines a labelling of $\mathscr{C}$ by composition. Now, we can identify $\mathscr{C}$ with the complete 


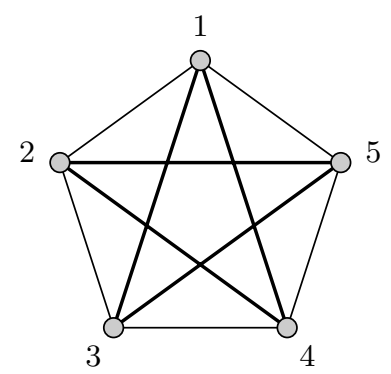

Figure 1: $G_{1}=12345 ; G_{2}=13524$.

graph on $\{1,2,3,4,5\}$ (see Fig. 1 ), where the edges of the cycle $G_{1}$ corresponding to $\Gamma_{1}$ are the edges of the pentagon while the edges of the other cycle $G_{2}$ corresponding to $\Gamma_{2}$ are the edges of the pentagram.

Now, observe that each clique $\mathscr{C}$ is determined by an isomorphism of $G_{2}$ onto $\Gamma_{2}$ and vice versa. These isomorphisms can be expressed in terms of appropriate labellings of $\Gamma_{2}$ : We write the cycle $G_{2}$ as a sequence $l_{1} l_{2} l_{3} l_{4} l_{5}$ and define the corresponding labelling

$$
\left[a_{2, k}, a_{2, k+1}\right] \mapsto l_{k+1} \text { for } k=0, \ldots, 3 \text { and }\left[a_{1,1}, a_{1,3}\right] \mapsto l_{5} \text {. }
$$

We have ten sequences corresponding to $G_{2}$ : 13524, 35241, 52413, 24135, 41352, 14253, 42531, 25314, 53142, 31425. Thus, we have ten cliques, which are depicted in Figure 2. Each clique is labelled by an appropriate sequence.

REMARK 5. Modulo permutation and/or reversal of the two coordinates, we have essentially two different types of 5-cliques in $\mathscr{I}^{2}$. They are exemplified by cliques with labels 24135 and 35241. Both types were previously mentioned in [14, Figure 27]. In Section 6, we discuss a combinatorial equivalence of cliques. As is easily seen, all 5-cliques are combinatorially equivalent.

\section{$5 \quad$ Maximum cliques in $\mathscr{I}^{3}$}

Our present goal is to describe all cliques of maximum cardinality in $\mathscr{I}^{3}$. We begin with a general discussion concerning cliques in $\mathscr{I}^{n}$.

Let us suppose that $\mathscr{G} \subset \mathscr{I}^{n}$ is a clique of cardinality $b_{n}$, where $n \geqslant 2$. Let $\mathscr{G}_{i}=$ $\left\{J_{i}: J \in \mathscr{G}\right\}, i \in[n]$. Let $s_{i}=s\left(\mathscr{G}_{i}\right)$ (see Proposition 6) and $f_{i}$ be a homomorphism of $\mathscr{G}_{i}$ into $\mathscr{I}\left(s_{i}\right)$. Let us set $f=f_{1} \times \cdots \times f_{n}$ and

$$
\mathscr{C}=f(\mathscr{G})=\left\{f_{1}\left(J_{1}\right) \times \cdots \times f_{n}\left(J_{n}\right): J \in \mathscr{G}\right\} .
$$

Clearly, $\mathscr{C}$ is a clique of maximum cardinality $b_{n}$ in $\mathscr{I}^{n}$. Let $\gamma_{i}=\sum_{I \in \mathscr{C}} I_{i}$. By the definition of $\mathscr{C}$, the formal sum $\gamma_{i} \in \mathbb{N} \mathscr{I}\left(s_{i}\right)$ is incompressible. Therefore, by Propositions 7 , and 9 ,

$$
s_{i} \leqslant 2 \alpha\left(\gamma_{i}\right)-1 \leqslant 2 b_{n-1}-1
$$



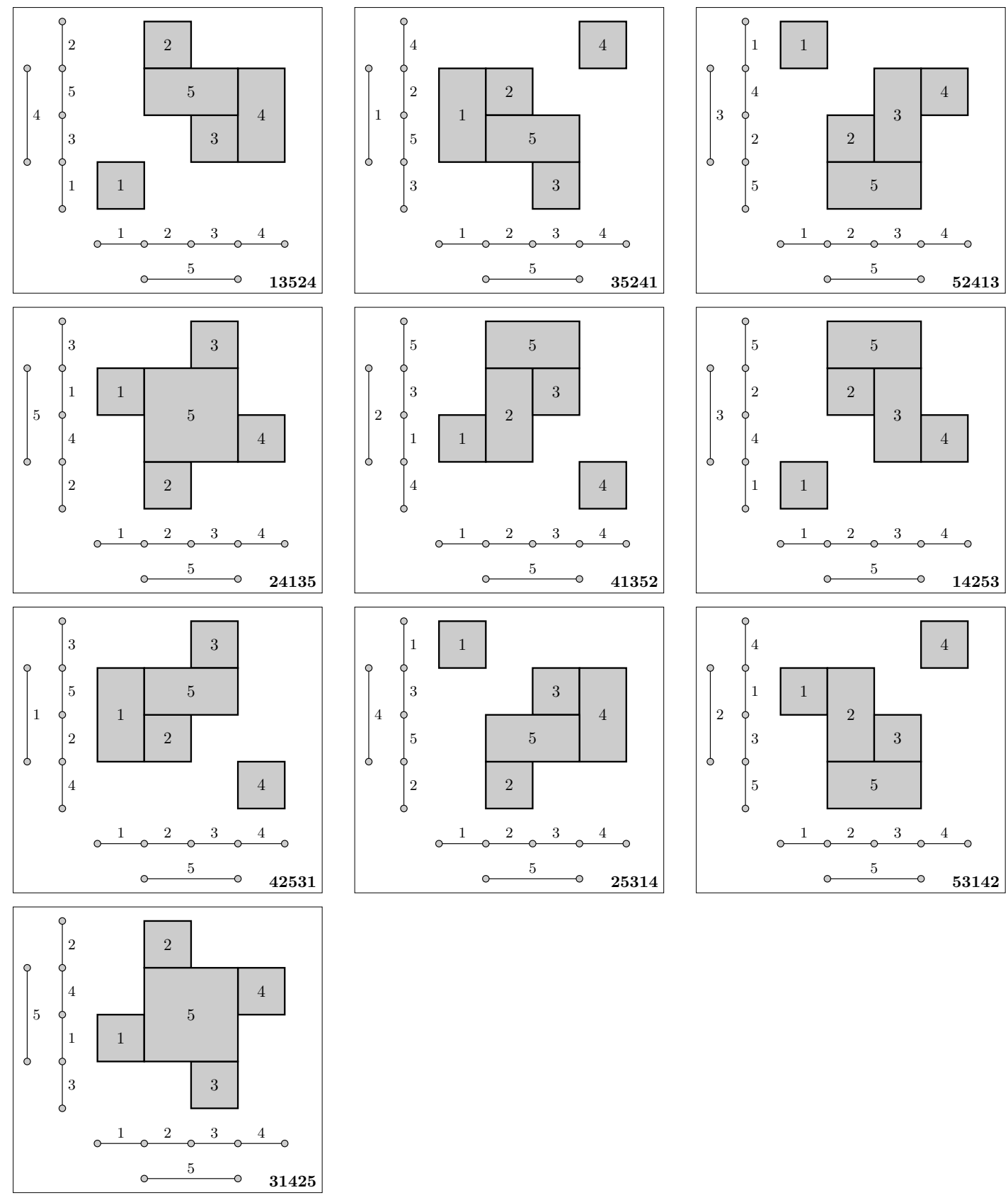

Figure 2: Cliques solving the construction problem for given $\Gamma_{1}$ and $\Gamma_{2}$. 
And if $s_{i} \geqslant 3$, then again by Proposition 7 ,

$$
\mathscr{S}\left(s_{i}\right) \subseteq \operatorname{supp} \gamma_{i}
$$

Let us remark that a description of cliques like $\mathscr{C}$, call them incompressible, is a strictly combinatorial problem: All such cliques consist of intervals $I$ contained in $\left[0,2 b_{n-1}-1\right]^{n}$ whose vertices have integer coordinates. Suppose that this problem can be effectively solved. As we have observed, if $\mathscr{G}$ is a clique of maximum cardinality, then there is a mapping $f$ which sends it to an incompressible clique. We can hope that this fact can be employed in order to give a full description of all maximum cliques in $\mathscr{I}^{n}$. As we shall see, this general strategy works for $n=3$.

EXAmple 1. Let $\mathscr{C} \subset \mathscr{I}^{3}$ consists of the following intervals:

$$
\begin{array}{cc}
1 . & {[0,1] \times[3,4] \times[1,4]} \\
2 . & {[0,1] \times[4,5] \times[2,4]} \\
3 . & {[1,2] \times[3,4] \times[1,3]} \\
4 . & {[1,2] \times[4,5] \times[2,3]} \\
5 . & {[1,3] \times[1,2] \times[3,4]} \\
6 . & {[1,4] \times[0,1] \times[3,4]} \\
7 . & {[2,3] \times[1,2] \times[4,5]} \\
8 . & {[2,4] \times[0,1] \times[4,5]} \\
9 . & {[3,4] \times[1,3] \times[1,2]} \\
10 . & {[3,4] \times[1,4] \times[0,1]} \\
11 . & {[4,5] \times[2,3] \times[1,2]} \\
12 . & {[4,5] \times[2,4] \times[0,1]}
\end{array}
$$

One can easily check that $\mathscr{C}$ is an incompressible clique in $\mathscr{I}^{3}$; that is, each $\gamma_{i}=\sum_{I \in \mathscr{C}} I_{i}$ is incompressible (in fact, $\gamma_{1}=\gamma_{2}=\gamma_{3}$ ). As $|\mathscr{C}|=12$, it follows that $b_{3} \geqslant 12$.

Now, we distinguish a set $L$ of combinations $\lambda \in \mathbb{N} \mathscr{I}^{1}$ such that for every incompressible clique $\mathscr{C}$ in $\mathscr{I}^{3}$ of the maximal cardinality, each $\gamma_{i}=\sum_{I \in \mathscr{C}} I_{i}$ is a member of $L$. This set is characterized by the following conditions:
(A) $12 \leqslant|\lambda| \leqslant 17$
(B) $\alpha(\lambda) \leqslant 5$;
(C) there is $s, 3 \leqslant s \leqslant 9$, such that $\mathscr{S}(s) \subseteq \operatorname{supp} \lambda \subseteq \mathscr{I}(s)$.

Condition (A) reflects the fact that $12 \leqslant b_{3} \leqslant 17$ (the second inequality is derived from Proposition 10) ; (B) is implied by Proposition 9 and the equality $b_{2}=5$. Condition (C) reflects (3) and (4). Only the assumption $s \geqslant 3$ requires clarification. Suppose $\lambda=\gamma_{1}$, where $\gamma_{1}=\sum_{I \in \mathscr{C}} I_{1}$ and $\mathscr{C}$ is an incompressible clique of cardinality $b_{3}$ in $\mathscr{I}^{3}$. If there would be $s=s_{1} \leqslant 2$, then

$$
12 \leqslant|\mathscr{C}|=\left|\gamma_{1}\right| \leqslant 2 \alpha\left(\gamma_{1}\right) \leqslant 10
$$




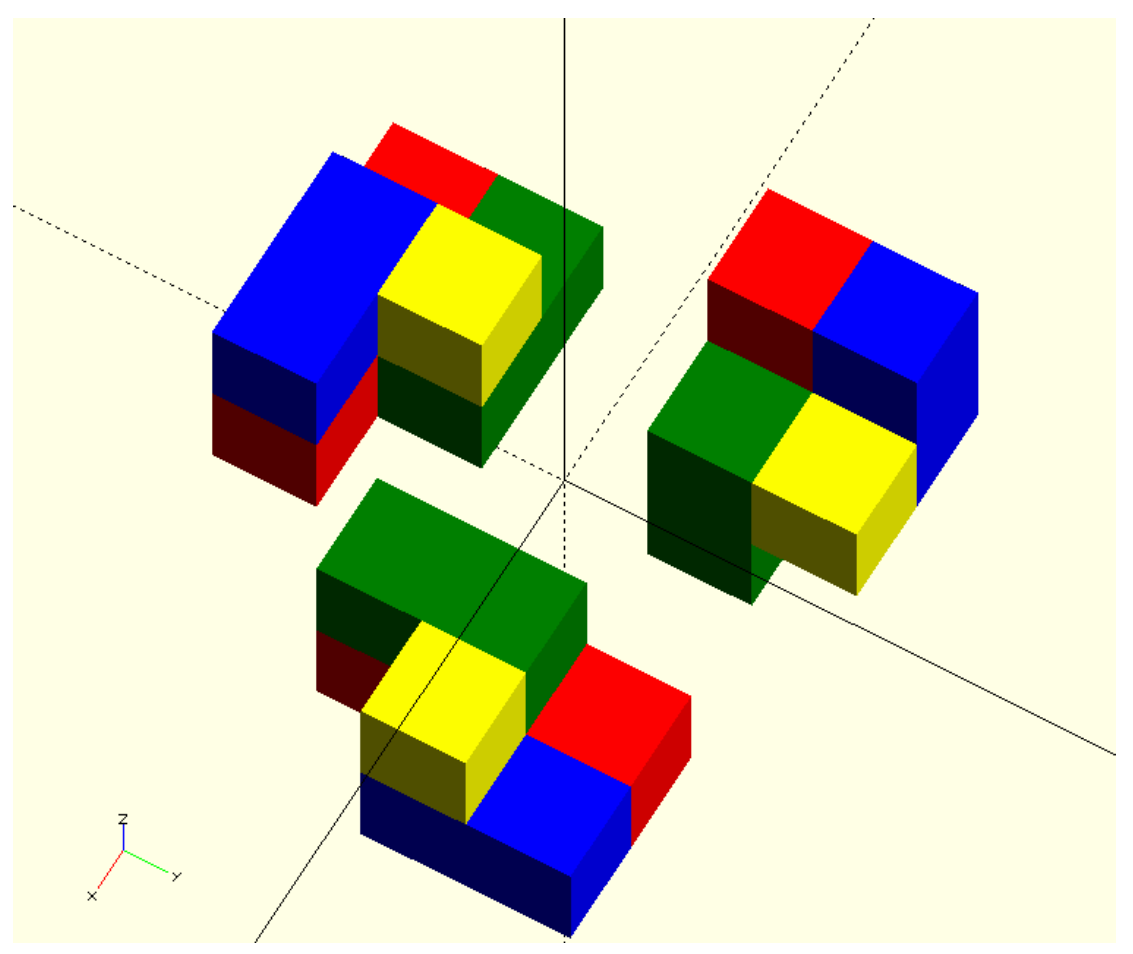

Figure 3: An illustration of the clique $\mathscr{C}$ described in Example 1. The clique is translated so that the circumscribed box is centred at the origin.

which is impossible.

If one wants to determine all members of $L$, then one needs an effective method to verify $(\mathrm{B})$. We discuss this question now.

Let $s$ be a positive integer. For $\varepsilon \in\{0,1\}^{s}$, let the subfamily $\mathscr{I}(s, \varepsilon)$ of $\mathscr{I}(s)$ be defined as follows

$$
\begin{aligned}
& {[u, v] \in \mathscr{I}(s, \varepsilon) \text { if and only if }[u, v] \in \mathscr{I}(s) \text { and either } 1 \leqslant u<v \leqslant s} \\
& \varepsilon_{u}=0, \varepsilon_{v}=1, \text { or }[u, v]=[0,1], \varepsilon_{1}=1, \text { or }[u, v]=[s, s+1], \varepsilon_{s}=0 .
\end{aligned}
$$

It is clear that all the families $\mathscr{I}(s, \varepsilon), \varepsilon \in\{0,1\}^{s}$, are independent sets with respect to the adjacency defined in $\mathscr{I}^{1}$. Moreover, each maximal independent subset of $\mathscr{I}(s)$ can be found among these families. This observation leads to the following

Proposition 12. Let $s$ be a positive integer. If $\lambda=\sum k_{I} I$ belongs $\mathbb{N} \mathscr{I}(s)$, then

$$
\alpha(\lambda)=\max \left\{\sum_{I \in \mathscr{I}(s, \varepsilon)} k_{I}: \varepsilon \in\{0,1\}^{s}\right\}
$$

This proposition enables us to identify the problem of determining $L$ as a problem of integer linear programming.

Let $L(s, v)$ be the set of all these $\lambda \in L$ for which $\mathscr{S}(s) \subseteq \operatorname{supp} \lambda \subseteq \mathscr{I}(s)$ (comp. $(\mathrm{C}))$ and $|\lambda|=v$. Clearly, $L$ is a union of the sets $L(s, v), 3 \leqslant s \leqslant 9,12 \leqslant v \leqslant 17$. A 
combination $\lambda=\sum_{I \in \mathscr{I}(s)} k_{I} I$ is a member of $L(s, v)$ if and only if $k_{I}, I \in \mathscr{I}(s)$, satisfy the following system of linear inequalities:

$$
\begin{aligned}
\sum_{I \in \mathscr{I}(s)} k_{I} & =v ; \\
\sum_{I \in \mathscr{I}(s, \varepsilon)} k_{I} & \leqslant 5, \text { for every } \varepsilon \in\{0,1\}^{s} ; \\
k_{I} & \geqslant 1, \text { for } I \in \mathscr{S}(s) .
\end{aligned}
$$

All solutions of this system can be found using standard mathematical packages. We have preferred to run a simple Python code, and to perform tests using SCIP, a mixed integer programming solver. As we have computed, $L(s, 14)$ is non-empty only for $s=9$. Moreover, $L(9,14)$ consists of a single element

$\bar{\lambda}=[0,1]+[1,2]+[2,3]+[3,4]+[4,5]+[5,6]+[6,7]+[7,8]+[8,9]+[9,10]+[1,3]+[3,5]+[5,7]+[7,9]$.

The sets $L(s, v)$, for $v>14$, appear to be empty, which can also be easily deduced from the uniqueness of $\bar{\lambda}$.

The following proposition is an immediate consequence of the definition of adjacency in $\mathscr{I}^{n}$.

Proposition 13. Let $\mathscr{C}$ be a subset of $\mathscr{I}^{n}$ and $\gamma_{i}=\sum_{I \in \mathscr{C}} I_{i}, i \in[n]$, then

$$
|E(\mathscr{C})| \leqslant \sum_{i=1}^{n}\left|E\left(\gamma_{i}\right)\right| .
$$

\section{Corollary 14.}

$$
b_{3} \leqslant 13
$$

Proof. Suppose that there is a clique in $\mathscr{I}^{3}$ which has 14 elements. Then there is an incompressible clique $\mathscr{C}$ of the same cardinality. By the uniqueness of $\bar{\lambda}$, we have $\bar{\lambda}=$ $\sum_{I \in \mathscr{C}} I_{i}$ for $i \in[3]$. One can check by hand that $|E(\bar{\lambda})|=20$. Now, by Proposition 13

$$
91=|E(\mathscr{C})| \leqslant 3|E(\bar{\lambda})|=60
$$

which is a contradiction.

It follows from our corollary that only the cases $v=12,13$ are meaningful in our quest for maximum cliques. The cardinalities of the sets $L(s, v), 3 \leqslant s \leqslant 9,12 \leqslant v \leqslant 13$, are collected in Table 1.

Let $\operatorname{Aut}(s)$ be the automorphism group of the graph $\mathscr{I}(s)$. By the correspondence $\operatorname{Aut}(s) \ni f \mapsto f_{*}$, it acts on $\mathbb{N} \mathscr{I}(s)$ in a natural way. Therefore, for every $L(s, v)$, one can form the quotient set $L(s, v) / \operatorname{Aut}(s)$. Let $\hat{L}(s, v)$ be a system of representatives (a selection) of $L(s, v) / \operatorname{Aut}(s)$. Let $L(v)$ be the union of all $L(s, v), 3 \leqslant s \leqslant 9$, for $v=12,13$, and $\hat{L}(v)$ be the union of the corresponding sets $\hat{L}(s, v)$. It should be clear that any maximum incompressible clique $\mathscr{D}$ of $\mathscr{I}^{3}$ can be recovered (by an automorphism) from a 


\begin{tabular}{|c|c|c|c|c|c|c|c|}
\hline$v$ & 3 & 4 & 5 & 6 & 7 & 8 & 9 \\
\hline 12 & 5 & 19 & 210 & 164 & 82 & 13 & 1 \\
13 & 0 & 0 & 20 & 35 & 55 & 13 & 3 \\
\hline
\end{tabular}

Table 1: Cardinality of $L(s, v)$.

\begin{tabular}{|l|c|c|c|c|c|c|c|}
\hline \multicolumn{1}{r|}{$v^{s}$} & 3 & 4 & 5 & 6 & 7 & 8 & 9 \\
\hline 12 & 1 & 4 & 37 & 29 & 21 & 5 & 1 \\
13 & 0 & 0 & 5 & 8 & 13 & 5 & 2 \\
\hline
\end{tabular}

Table 2: Cardinality of $\hat{L}(s, v)$.

clique $\mathscr{C}$ such that $\gamma_{i}=\sum_{I \in \mathscr{C}} I_{i} \in \hat{L}(v)$, for $i \in[3]$, where $v=|\mathscr{D}|$. Therefore, we may always consider $\hat{L}(v)$ instead of $L(v)$. This will slightly simplify further considerations, as in general the sets $\hat{L}(s, v)$ are smaller than $L(s, v)$. In order to fix $\hat{L}(v)$ we work with, we collect all the sets $\hat{L}(s, v)$ in Appendix B. We present in Table 2 all the cardinalities of $\hat{L}(s, v)$ to allow the reader to compare them with the corresponding cardinalities gathered in Table 1.

We adopt a strategy similar to the one we have applied to find maximum cliques in $\mathscr{I}^{2}$. We begin with solving the following 'restricted' construction problem as a step towards the classification of all maximum cliques in $\mathscr{I}^{3}$ :

For every triple $\vec{\gamma}=\left(\gamma_{1}, \gamma_{2}, \gamma_{3}\right)$ in $\hat{L}(v)^{3}$, where $v=12,13$, find all cliques $\mathscr{C}$ in $\mathscr{I}^{3}$ such that $\gamma_{i}=\sum_{I \in \mathscr{C}} I_{i}$ for every $i \in[3]$.

Let us define the sets $N(v)$ for $v=12,13$ :

$$
N(v)=\left\{\vec{\gamma} \in \hat{L}(v)^{3}: E\left(\gamma_{1}\right)+E\left(\gamma_{2}\right)+E\left(\gamma_{3}\right) \geqslant\left(\begin{array}{l}
v \\
2
\end{array}\right)\right\} .
$$

Proposition 13 shows that if the construction problem has a solution for $\vec{\gamma} \in \hat{L}(v)^{3}$, then $\vec{\gamma} \in N(v)$. Therefore, we can consider our problem only for elements of the sets $N(v)$. We can make further restrictions by selecting a triple from each class of triples equivalent up to reordering of components. However, we want to make our choice somewhat special.

We may distinguish between two types of $\lambda \in \hat{L}(v)$ :

(I) There are induced combinations $\beta^{1}$ and $\beta^{2}$ of $\lambda$ (see Introduction for the definition) such that

$$
\left|\beta^{j}\right|=\alpha\left(\beta^{j}\right)=5 \text {, for every } j \in[2],
$$

(I. 2) $\operatorname{supp} \beta^{1} \cap \operatorname{supp} \beta^{2}=\emptyset$. 
(II) $\lambda$ is not of type I.

Let us remark that (I. 1$)$ means that $G_{\beta^{j}}$ are 5 -anticliques. As it can be easily computed (see Appendix B), each $\lambda$ of type II satisfies the inequality

$$
E(\lambda) \leqslant \frac{1}{3}\left(\begin{array}{l}
v \\
2
\end{array}\right)
$$

Moreover, this inequality is sharp with only one exception; that is,

$$
\lambda^{*}=[0,1]+[1,2]+[1,3]+[1,5]+[2,3]+[2,5]+[3,4]+2[3,5]+[4,5]+2[5,6] .
$$

(Clearly, $\lambda^{*}$ belongs to $\hat{L}(5,12)$ then). As a consequence, there is only one $\vec{\gamma} \in N(v)$ such that all $\gamma_{i}$ are of type II, in which case they are equal to $\lambda^{*}$. The following result shows that this particular $\vec{\gamma}$ can be excluded from further considerations.

Proposition 15. The construction problem has no solutions for $\vec{\gamma}=\left(\lambda^{*}, \lambda^{*}, \lambda^{*}\right)$.

Proof. Conversely, suppose there is a clique $\mathscr{C}$ in $\mathscr{I}^{3}$ such that $\lambda^{*}=\sum_{I \in \mathscr{C}} I_{i}$, whenever $i \in[3]$. As $[5,6] \in \operatorname{supp} \lambda^{*}$, there is $K \in \mathscr{C}$ for which $K_{1}=[5,6]$. Similarly, there are $I^{k} \in \mathscr{C}$ such that $I_{1}^{k}=[k, 5]$, for $k \in[4]$. Since [3,5] occurs with multiplicity 2 in $\lambda^{*}$, there is $J^{3} \in \mathscr{C}$ which is different from $I^{3}$, and satisfies the equation $J_{1}^{3}=[3,5]$. Since $\left|E\left(\lambda^{*}\right)\right|=22$ and $|E(\mathscr{C})|=66$, it follows from the definition of adjacency in $\mathscr{I}^{3}$ and Proposition 13 that for every pair $C, D \in \mathscr{C}$ there is only one $i \in[3]$ for which the intervals $C_{i}$ and $D_{i}$ are adjacent (i.e. intersect at a single point). Since all $I_{1}^{k}, k \in[4]$, form an anticlique, we deduce that $\mathscr{B}=\left\{I_{\{1\}^{c}}^{k}: k \in[4]\right\} \cup\left\{J_{\{1\}^{c}}^{3}\right\}$ is a 5 -clique in $\mathscr{I}^{2}$. Therefore, as is shown in Section 4, the families $\mathscr{B}_{i}=\left\{I_{i}^{k}: k \in[4]\right\} \cup\left\{J_{i}^{3}\right\}, i=2,3$, have to be 5 -cycles contained in supp $\lambda^{*}$. Let us collect all possible 5-cycles with vertices in $\operatorname{supp} \lambda^{*}$ :

$$
\begin{array}{ll}
\text { 1. } & {[0,1],[1,2],[2,3],[3,4],[1,3]} \\
2 . & {[0,1],[1,2],[2,5],[5,6],[1,5]} \\
3 . & {[2,3],[3,4],[4,5],[5,6],[3,5]} \\
\text { 4. } & {[0,1],[1,3],[3,5],[5,6],[1,5]} \\
\text { 5. } & {[1,2],[2,3],[3,5],[5,6],[2,5]}
\end{array}
$$

As $K_{1}$ is adjacent to each of the intervals $I_{1}^{k}, k \in[4]$, interval $K_{i}$ cannot be adjacent to any of the intervals belonging to $\mathscr{B}_{i}$, for $i=2,3$. Consequently, if a 5 -cycle among listed were equal to $\mathscr{B}_{i}$, then there would be an interval in supp $\lambda^{*}$ which is not adjacent to any member of this cycle. Cycles no. 4 and no. 5 do not conform this condition, and as such can be eliminated. On the other hand, for cycle no. 1 the only existing interval is $[5,6]$, for cycle no. 2 , the interval $[3,4]$ and for cycle no. 3 , the interval $[0,1]$. Thus, $\left\{K_{2}, K_{3}\right\} \subset\{[0,1],[3,4],[5,6]\}$. Let us remind that $[5,6]$, similarly as $[3,5]$, occurs in $\lambda^{*}$ with multiplicity 2. Therefore, there is $L \in \mathscr{C} \backslash\{K\}$ such that $L_{1}=[5,6]$. By the same argument as applied to $K,\left\{L_{2}, L_{3}\right\} \subset\{[0,1],[3,4],[5,6]\}$. Since $[0,1],[3,4],[5,6]$ form an anticlique, $K$ and $L$ are not adjacent, which contradicts the assumption that $\mathscr{C}$ is a clique. 
Therefore, by Proposition 15, we may assume that at least one of the components of $\vec{\gamma} \in N(v)$ is of type I. We may declare that $\gamma_{3}$ is such a component. Suppose there is a clique $\mathscr{C} \subset \mathscr{I}^{3}$ of cardinality $v$ to be guessed such that $\gamma_{i}=\sum_{I \in \mathscr{C}} I_{i}$. Let $\beta^{j}, j \in[2]$, be combinations induced from $\gamma_{3}$, as described in the definition of type I. Since these combinations have disjoint supports there are disjoint subfamilies $\mathscr{C}^{j}, j \in[2]$, of $\mathscr{C}$ such that $\beta^{j}=\sum_{I \in \mathscr{C}^{j}} I_{3}$. Since $\beta^{j}$ are 5-anticliques, $\mathscr{C}_{\{1,2\}}^{j}=\left\{I_{\{1,2\}}: I \in \mathscr{C}^{j}\right\}$ have to be 5-cliques in $\mathscr{I}^{2}$. Thus, the families $\mathscr{C}_{i}^{j}=\left\{I_{i}: I \in \mathscr{C}^{j}\right\}, i, j \in[2]$, are 5-cycles, as is explained in Section 4.

Let us write

$$
\gamma=\sum_{I \in \mathscr{C}} I, \quad \gamma^{j}=\sum_{I \in \mathscr{C}^{j}} I
$$

Clearly, $\gamma \geqslant \gamma^{1}+\gamma^{2}$. Therefore, there is $\gamma^{3} \in \mathbb{N} \mathscr{I}^{3}$ such that $\gamma=\sum_{j=1}^{3} \gamma^{j}$ and $\left|\gamma^{3}\right|=v-10$. Consequently,

$$
\gamma_{\{1,2\}}=\sum_{j=1}^{3} \gamma_{\{1,2\}}^{j} \quad \text { and } \quad \gamma_{i}=\sum_{j=1}^{3} \gamma_{i}^{j} \quad \text { for } i \in[2],
$$

where each $\gamma_{i}^{j}, j \in[2]$, is the formal sum of all intervals constituting the 5 -cycle $\mathscr{C}_{i}^{j}$. Moreover, by Corollary 5

$$
\alpha\left(\gamma_{\{1,2\}}\right) \leqslant \omega\left(\gamma_{3}\right) \leqslant \omega\left(\mathscr{I}^{1}\right) \leqslant 2 .
$$

Now, we are prepared to establish a procedure for finding all incompressible cliques of maximum cardinality in $\mathscr{I}^{3}$. Since each $\lambda \in \hat{L}(v)$ can potentially be equal to $\gamma_{1}$ or $\gamma_{2}$ for a certain maximum clique in $\mathscr{C}$, we have to produce all possible decompositions $\lambda=\sum_{j=1}^{3} \lambda^{j}$, as described in the second part of (8). In other words, we have to extract all possible quadruples $q=\left(\lambda^{1}, \lambda^{2}, \lambda^{3}, \lambda\right)$ such that $\lambda^{j}$ are 5-cycles for $j \in[2]$ and $\sum_{j=1}^{3} \lambda^{j}$ equals $\lambda$. To this end, we need to determine all 5 -cycles with their supports contained in $\mathscr{I}(9)$.

Step 1. Find the set $\mathrm{Co}_{5}$ consisting of all 5-cycles contained in $\mathbb{N} \mathscr{I}(9)$.

Let us recall (see Section 4) that there is a one-to-one correspondence between the sets of all 5 -cycles in $\mathbb{N} \mathscr{I}(9)$, and in $\mathscr{I}(9)$ given by the mapping $\kappa \mapsto \operatorname{supp} \kappa$. From a technical point of view, the latter set is calculated rather than $\mathrm{Co}_{5}$.

The first two components of the quadruples under consideration are 5-cycles, therefore, it seems reasonable to determine the Cartesian product $\mathrm{Co}_{5} \times \mathrm{Co}_{5}$. Since $\left|\mathrm{Co}_{5}\right|=118$, the Cartesian square of $\mathrm{CO}_{5}$ has 13924 elements. Observe however that not all pairs of 5-cycles can be components of a quadruple. For example, if $\Gamma=[0,1]+[1,2]+[2,3]+[3,4]+[1,3]$ and $\Delta=[0,1]+[1,3]+[3,7]+[7,8]+[1,7]$ would be such components for $v=12$, then there would exist $\lambda \in \hat{L}(12)$ such that $\Gamma+\Delta \leqslant \lambda$. If we take into account (C), the support of such a $\lambda$ has to contain one of the two sets $A=\mathscr{S}(8)$ or $A=\mathscr{S}(9)$, which is impossible as $A-(\operatorname{supp}(\Gamma) \cup \operatorname{supp}(\Delta))$ has more than two elements. To define the set of appropriate pairs of 5 -cycles, we need the function $\Gamma \mapsto \operatorname{sep}(\Gamma)$ which determines the smallest $s$ such 
that $\operatorname{supp}(\Gamma) \subset \mathscr{I}(s)$. As $\Gamma$ can be written as a combination $\left[a_{0}, a_{1}\right]+\left[a_{1}, a_{2}\right]+\left[a_{2}, a_{3}\right]+$ $\left[a_{3}, a_{4}\right]+\left[a_{1}, a_{3}\right]$, where $a_{0}<\ldots<a_{4}$, and all $\left[a_{i}, a_{j}\right]$ are in $\mathscr{I}(9)$, we have

$$
\operatorname{sep}(\Gamma)= \begin{cases}a_{3}, & \text { if } a_{4}=a_{3}+1 \\ a_{4}, & \text { otherwise }\end{cases}
$$

Step 2. For $v=12,13$, find the set $\mathrm{Cp}_{5}(v)$, consisting of all pairs $(\Gamma, \Delta) \in \mathrm{Co}_{5} \times \mathrm{Co}_{5}$ such that $|\mathscr{S}(s)-(\operatorname{supp}(\Gamma) \cup \operatorname{supp}(\Delta))| \leqslant v-10$, where $s=\max \{\operatorname{sep}(\Gamma), \operatorname{sep}(\Delta)\}$.

The sets $\mathrm{Cp}_{5}(v)$ are much smaller compared with $\mathrm{Co}_{5} \times \mathrm{Co}_{5}$, and yet $\left(\lambda^{1}, \lambda^{2}\right)$ belongs to $\mathrm{Cp}_{5}(v)$ for every admissible quadruple $q=\left(\lambda^{1}, \lambda^{2}, \lambda^{3}, \lambda\right)$. We have $\left|\mathrm{Cp}_{5}(12)\right|=384$ and $\left|\mathrm{Cp}_{5}(13)\right|=1135$.

Step 3. For $v=12,13$, find the set $\operatorname{Mc}(v)$ of all quadruples $\left(\lambda^{1}, \lambda^{2}, \lambda^{3}, \lambda\right)$ such that $\left(\lambda^{1}, \lambda^{2}\right) \in \mathrm{Cp}_{5}(v), \lambda \in \hat{L}(v), \lambda^{1}+\lambda^{2} \leqslant \lambda$ and $\lambda^{3}=\lambda-\lambda^{1}-\lambda^{2}$.

The cardinalities of the sets $\operatorname{Mc}(v)$ are as follows: $|\operatorname{Mc}(12)|=372,|\operatorname{Mc}(13)|=409$.

Step 4. Construct all candidates for $\gamma_{\{1,2\}}$.

We want them to be decomposed as described in (8), and to satisfy (9). To this end, for every pair $q=\left(\lambda^{1}, \lambda^{2}, \lambda^{3}, \lambda\right), \bar{q}=\left(\bar{\lambda}^{1}, \bar{\lambda}^{2}, \bar{\lambda}^{3}, \bar{\lambda}\right)$ belonging to $\operatorname{Mc}(v)$, let us define the set $\operatorname{Fl}(q, \bar{q})$ of all quadruples $p=\left(\varphi^{1}, \varphi^{2}, \varphi^{3}, \varphi\right) \in\left(\mathbb{N} \mathscr{I}^{2}\right)^{4}$ such that

(F1) $G_{\varphi^{j}}$ are 5-cliques in $\mathscr{I}^{2}$, for $j \in[2]$,

(F2) $\varphi=\varphi^{1}+\varphi^{2}+\varphi^{3}$,

(F3) if $p_{i}=\left(\varphi_{i}^{1}, \varphi_{i}^{2}, \varphi_{i}^{3}, \varphi_{i}\right), i \in[2]$, then $p_{1}=q, p_{2}=\bar{q}$;

(F4) $\alpha(\varphi) \leqslant 2$.

As is stated in Sect. 4, there are 10 ways to combine two 5 -cycles from $\mathscr{I}$ to get a 5 -clique in $\mathscr{I}^{2}$. Moreover, there are at most $(v-10)$ ! ways to merge $\lambda^{3}$ with $\bar{\lambda}^{3}$ in order to get $\varphi^{3}$. Therefore, the cardinality of $\mathrm{Fl}(q, \bar{q})$ is at most $100(v-10) ! \leqslant 600$. Condition $(\mathrm{F} 4)$ can cut this last number substantially.

Step 5. Find the sets Flat $(v), v=12,13$, which are the unions of $\operatorname{Fl}(q, \bar{q})$, when $(q, \bar{q})$ runs over $\operatorname{Mc}(v)^{2}$.

It appears that Flat(13) is empty. Consequently, no 13-cliques in $\mathscr{I}^{\mathbf{3}}$ exist. By Example 1, we conclude:

Theorem 16.

$$
b_{3}=12
$$



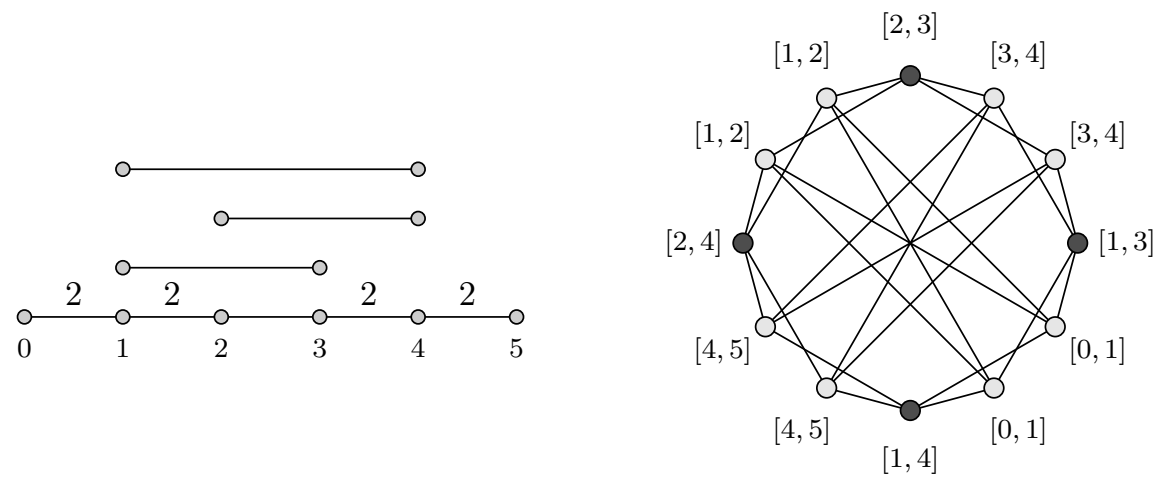

Figure 4: Diagrams for $\lambda^{\boldsymbol{*}}$.
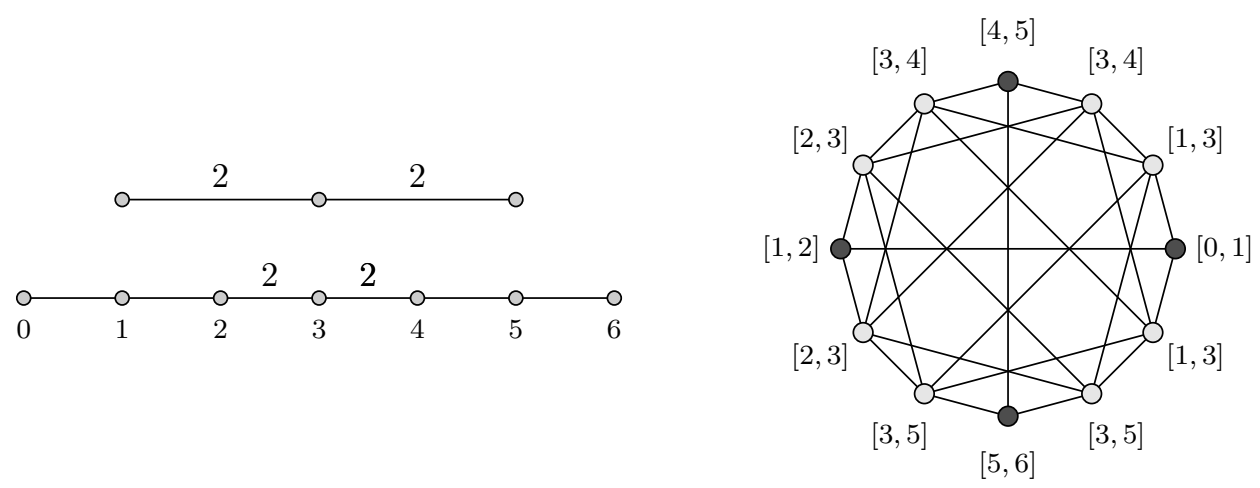

Figure 5: Diagrams for $\lambda^{\uparrow}$.

Flat(12) contains 96 elements. Interestingly enough, if $\left(\varphi^{1}, \varphi^{2}, \varphi^{3}, \varphi\right)$ belongs to Flat(12), then $\varphi_{1}=\varphi_{2}$. Moreover, $\varphi_{1}$ is equal to one of the three combinations:

$$
\begin{aligned}
\lambda^{\star}= & 2[0,1]+2[1,2]+[1,3]+[1,4]+[2,3]+[2,4]+2[3,4]+2[4,5], \\
\lambda^{\star}= & {[0,1]+[1,2]+2[1,3]+2[2,3]+2[3,4]+2[3,5]+[4,5]+[5,6], } \\
\lambda^{\diamond}= & {[0,1]+[1,2]+[1,3]+[2,3]+[3,4]+2[3,5]+[4,5]+[5,6] } \\
& +[5,7]+[6,7]+[7,8] .
\end{aligned}
$$

Figures 4-6 show self-explanatory diagrams for them. Combinations $\lambda^{\boldsymbol{*}}$ and $\lambda^{\boldsymbol{\omega}}$ are of type I, as exemplified by Table 3 , while $\lambda^{\diamond}$ is of type II.

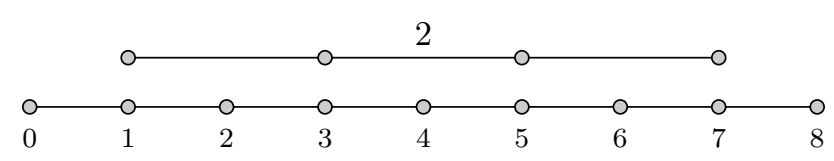

Figure 6: Diagram for $\lambda^{\diamond}$.

Proposition 17. If the construction problem has a solution for $\vec{\gamma}=\left(\gamma_{1}, \gamma_{2}, \gamma_{3}\right) \in N(12)$, then

$$
\vec{\gamma}=\left(\lambda^{\star}, \lambda^{\star}, \lambda^{\star}\right) \quad \text { or } \quad \vec{\gamma}=\left(\lambda^{\star}, \lambda^{\star}, \lambda^{\star}\right) .
$$


Proof. Let us remind that we may assume $\gamma_{3}$ to be of type I. Then it follows from the definition, and properties of Flat(12) that $\gamma_{1}=\gamma_{2}$ and $\gamma_{1} \in\left\{\lambda^{\boldsymbol{\bullet}}, \lambda^{\boldsymbol{\bullet}}, \lambda^{\diamond}\right\}$.

Suppose first that $\gamma_{1}=\lambda^{\diamond}$. Recall that $\left|E\left(\lambda^{\diamond}\right)\right|=19$. Since $\vec{\gamma} \in N(12)$, it can be deduced that $\left|E\left(\gamma_{3}\right)\right| \geqslant 28$. There is a unique element in $\hat{L}(12)$ which satisfies this inequality. Namely, $\gamma_{3}=2[0,1]+2[1,2]+3[1,3]+2[2,3]+3[3,4]$. $\left(\left|E\left(\gamma_{3}\right)\right|=29\right.$, to be precise). Suppose that there is a 12 -clique $\mathscr{C}$ such that $\gamma_{i}=\sum_{I \in \mathscr{C}} I_{i}, i \in[3]$. Let $\beta^{1}=$ $2[0,1]+3[3,4], \beta^{2}=2[1,2]+3[3,4]$ and $\beta^{3}=3[1,3]+2[2,3]$. As all $\beta^{j}$ are subcombinations of $\gamma_{3}$, there are $\gamma^{j} \leqslant \gamma=\sum_{I \in \mathscr{C}} I$ for which $\gamma_{3}^{j}=\beta^{j}$. Observe now that $\alpha\left(\beta^{j}\right)=5$ for each $\beta^{j}$. We already know that it implies each $\gamma_{1}^{j}$ to be a 5 -cycle. Since combinations $\beta^{1}, \beta^{3}$ have disjoint supports, we deduce that $\gamma_{1}^{1}+\gamma_{1}^{3} \leqslant \gamma_{1}=\lambda^{\diamond}$. There are only two 5-cycles such that their sum does not exceed $\lambda^{\diamond}$; these are $\kappa=[0,1]+[1,2]+[2,3]+[3,4]+[1,3]$ and $\kappa^{\prime}=[4,5]+[5,6]+[6,7]+[7,8]+[5,7]$. Thus, $\left\{\gamma_{1}^{1}, \gamma_{1}^{3}\right\}=\left\{\kappa, \kappa^{\prime}\right\}$. Similarly, by the fact that $\beta^{2}, \beta^{3}$ have disjoint supports, we obtain $\left\{\gamma_{1}^{2}, \gamma_{1}^{3}\right\}=\left\{\kappa, \kappa^{\prime}\right\}$. Since $\gamma_{1}=\lambda^{\diamond}$, there is $J \in \mathscr{C}$ such that $J_{1}=[3,5]$. Since the supports of $\beta^{j}, j \in[3]$ form a covering of supp $\gamma_{3}$, there is $k$ such that $J_{3} \in \operatorname{supp} \beta^{k}$. As $\beta^{k}$ is an induced subcombination of $\gamma_{3}$, we deduce that $J \in \operatorname{supp} \gamma^{k}$. Therefore,

$$
[3,5] \in \operatorname{supp} \gamma_{1}^{k} \subset \operatorname{supp} \kappa \cup \operatorname{supp} \kappa^{\prime}=\{[i, i+1]: i=0, \ldots, 7\} \cup\{[1,3],[5,7]\},
$$

which is a contradiction.

If $\gamma_{1} \in\left\{\lambda^{\boldsymbol{\omega}}, \lambda^{\boldsymbol{\omega}}\right\}$, then it is of type I. By symmetry, it enforces $\gamma_{2}=\gamma_{3}$. Thus $\vec{\gamma} \in\left\{\left(\lambda^{\boldsymbol{\omega}}, \lambda^{\boldsymbol{\omega}}, \lambda^{\boldsymbol{\omega}}\right),\left(\lambda^{\boldsymbol{\omega}}, \lambda^{\boldsymbol{\omega}}, \lambda^{\boldsymbol{\omega}}\right)\right\}$.

Now, the final steps of our construction follow. Let us extract two sets from Flat(12):

$$
\begin{aligned}
& \mathrm{Fl}^{\boldsymbol{\alpha}}=\left\{\left(\varphi^{1}, \varphi^{2}, \varphi^{3}\right):\left(\varphi^{1}, \varphi^{2}, \varphi^{3}, \varphi\right) \in \operatorname{Flat}(12), \varphi_{1}=\lambda^{\boldsymbol{中}}\right\} \\
& \mathrm{Fl}^{\boldsymbol{\aleph}}=\left\{\left(\varphi^{1}, \varphi^{2}, \varphi^{3}\right):\left(\varphi^{1}, \varphi^{2}, \varphi^{3}, \varphi\right) \in \operatorname{Flat}(12), \varphi_{1}=\lambda^{\boldsymbol{\bullet}}\right\} .
\end{aligned}
$$

Let $\left(\beta^{1}, \beta^{2}, \beta^{3}\right)$ be defined so that $\lambda^{\boldsymbol{*}}=\beta^{1}+\beta^{2}+\beta^{3}$ where $\beta^{1}, \beta^{2}$ are the elements of $\lambda^{\text {* }}$-row of Table 3 .

Step 5 Find

$\mathrm{Cq}^{\boldsymbol{\mu}}=\left\{\pi^{1}+\pi^{2}+\pi^{3} \in \mathbb{N}^{3}:\left(\pi_{1}^{1}, \pi_{1}^{2}, \pi_{1}^{3}\right) \in \mathrm{Fl}^{\boldsymbol{\mu}},\left(\pi_{2}^{1}, \pi_{2}^{2}, \pi_{2}^{3}\right)=\left(\beta^{1}, \beta^{2}, \beta^{3}\right), \alpha\left(\pi^{1}+\pi^{2}+\pi^{3}\right)=1\right\}$.

\begin{tabular}{|c|c|c|}
\hline & $\beta^{1}$ & $\beta^{2}$ \\
\hline$\lambda^{\boldsymbol{中}}$ & $2[0,1]+[2,4]+2[3,4]$ & $2[1,2]+[1,3]+2[4,5]$ \\
$\lambda^{\boldsymbol{\bullet}}$ & {$[0,1]+2[3,4]+2[3,5]$} & $2[1,3]+2[2,3]+[5,6]$ \\
\hline
\end{tabular}

Table 3: $\lambda^{\boldsymbol{n}}$ and $\lambda^{\boldsymbol{\omega}}$ are of type I. 
Therefore, $\mathrm{Cq}^{\boldsymbol{*}}$ consists of all 12-cliques $\mathscr{C} \subset \mathscr{I}^{3}$, written as formal combinations, such that $\sum_{I \in \mathscr{C}} I_{i}=\lambda^{\boldsymbol{*}}$ for $i \in[3]$. It appears that we have

$$
\left|\mathrm{Cq}^{\boldsymbol{\mu}}\right|=64
$$

In the same manner, we define $\mathrm{Cq}^{\star}$.

\section{Step 6 Find $\mathrm{Cq}^{\infty}$.}

The elements of $\mathrm{Cq}^{\uparrow}$ correspond to 12-cliques $\mathscr{C} \subset \mathscr{I}^{3}$ such that $\sum_{I \in \mathscr{C}} I_{i}=\lambda^{\star}$. We have

$$
\left|\mathrm{Cq}^{\boldsymbol{\omega}}\right|=256 \text {. }
$$

THEOREM 18. $\mathscr{C}$ is an incompressible maximum clique in $\mathscr{I}^{3}$ if and only if $\sum_{I \in \mathscr{C}} I \in$ $\mathrm{Cq} \cup \mathrm{Cq}^{\boldsymbol{*}}$.

Proof. $(\Rightarrow)$ Let $\gamma=\sum_{I \in \mathscr{C}} I$. By Theorem 16 and the definition of the sets $L(s, v)$, there are $s_{i}, i \in[3]$, such that $\gamma_{i} \in L\left(s_{i}, 12\right)$. Let automorphisms $f_{i} \in \operatorname{Aut}\left(s_{i}\right)$ be chosen so that $\left(f_{i}\right)_{*}\left(\gamma_{i}\right) \in \hat{L}\left(s_{i}, 12\right)$. Then $\left(\left(f_{1}\right)_{*}\left(\gamma_{1}\right),\left(f_{2}\right)_{*}\left(\gamma_{2}\right),\left(f_{3}\right)_{*}\left(\gamma_{3}\right)\right)$ has to belong to $N(12)$. By Proposition 17, $\left(f_{i}\right)_{*}\left(\gamma_{i}\right)=\lambda^{\boldsymbol{*}}$ for $i \in[3]$ or $\left(f_{i}\right)_{*}\left(\gamma_{i}\right)=\lambda^{*}$ for $i \in[3]$. Observe now that $\lambda^{\boldsymbol{*}}$ is invariant under the action of Aut(4); that is, if $g \in \operatorname{Aut}(4)$, then $g_{*}\left(\lambda^{\boldsymbol{*}}\right)=\lambda^{\boldsymbol{*}}$. Similarly, $\lambda$ is invariant under the action of Aut(5). Therefore, $\left(\gamma_{1}, \gamma_{2}, \gamma_{3}\right)$ is one of the

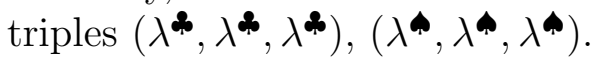

$(\Leftarrow)$ As each member of $L(s, v)$ satisfies only a necessary condition for being incompressible (compare (C) and Proposition 7), it may happen that $\lambda^{\boldsymbol{\omega}}$ or $\lambda^{\boldsymbol{\omega}}$ are compressible. By Proposition 17, the only possibility is that $\lambda^{\boldsymbol{n}}$ can be compressed to $\lambda^{\boldsymbol{\alpha}}$; that is, there is a homomorphism $h: \mathscr{I}(5) \rightarrow \mathscr{I}(4)$ such that $h_{*}\left(\lambda^{\boldsymbol{*}}\right)=\lambda^{\boldsymbol{*}}$. Consequently, $\left|E\left(\lambda^{\boldsymbol{*}}\right)\right| \geqslant$ $\left|E\left(\lambda^{\top}\right)\right|$. The latter is impossible, as $\left|E\left(\lambda^{\boldsymbol{*}}\right)\right|=24$ while $\left|E\left(\lambda^{\boldsymbol{*}}\right)\right|=26$.

\section{Isomorphic incompressible cliques. Automorphisms}

From now on, we shall interpret $\mathrm{Cq}^{\boldsymbol{\star}}$ and $\mathrm{Cq}^{\boldsymbol{\omega}}$ as families of 12-cliques rather than formal combinations of the 3-intervals these cliques consist of. As in the preceding section, let $\operatorname{Aut}(s)$ be the automorphism group of the graph $\mathscr{I}(s)$. Let $\mathrm{Aut}^{3}(s)$ consists of the product mappings $f=f_{1} \times f_{2} \times f_{3}$, where $f_{i} \in \operatorname{Aut}(s)$. It is clear that for every $\mathscr{C} \in \mathrm{Cq}^{\boldsymbol{*}}$ and $f \in \operatorname{Aut}^{3}(4)$, we have $f(\mathscr{C}) \in \mathrm{Cq}^{\boldsymbol{\alpha}}$. Therefore, the group $\mathrm{Aut}^{3}(4)$ acts on $\mathrm{Cq}^{\boldsymbol{\alpha}}$. It can be rather easily computed that $\mathrm{Cq}^{2}$ is an orbit of $\mathrm{Aut}^{3}(4)$. Consequently, we have:

Proposition 19. All cliques belonging to $\mathrm{Cq}^{\boldsymbol{\alpha}}$ are isomorphic.

Similarly, $\mathrm{Aut}^{3}(5)$ acts on $\mathrm{Cq}^{\uparrow}$. It appears that $\mathrm{Cq}^{\star}$ splits into 3 orbits under the action of $\operatorname{Aut}^{3}(5)$. (Two of them are of cardinality 64 while the remaining orbit is of cardinality 128). Clearly, it does not necessarily mean that there are three pairwise nonisomorphic cliques. In fact, they are not.

Let $S_{3}$ be the symmetry group of $\{1,2,3\}$. Each $\sigma \in S_{3}$ induces the isometry $s_{\sigma}$ of $\mathbb{R}^{3}$ which in turn extends to intervals: $s_{\sigma}(I)=I_{\sigma^{-1}(1)} \times I_{\sigma^{-1}(2)} \times I_{\sigma^{-1}(3)}$. Obviously, $s_{\sigma}$ 
defines an isomorphism of cliques. It is rather obvious that the composites $s_{\sigma} \circ f$, where $\sigma \in S_{3}$ and $f \in \operatorname{Aut}^{3}(s)$, form a group of isomorphisms of cliques. Algebraically, it is a semidirect product of $S_{3}$ and $\operatorname{Aut}^{3}(s)$. Let us denote it by $A^{3}(s)$. A computation shows that $\mathrm{Cq}$ splits into two orbits under the action of $A^{3}(5)$. Let us fix some representatives of these orbits for further discussion, and call them $\mathscr{D}^{1}$ and $\mathscr{D}^{2}$ (Table 4, Figure 6).

$$
\begin{array}{ccl}
1 . & {[0,1] \times[1,3] \times[2,3]} & {[0,1] \times[1,3] \times[2,3]} \\
2 . & {[1,2] \times[2,3] \times[1,3]} & {[1,2] \times[2,3] \times[1,3]} \\
3 . & {[1,3] \times[1,2] \times[2,3]} & {[1,3] \times[1,2] \times[2,3]} \\
4 . & {[1,3] \times[2,3] \times[0,1]} & {[1,3] \times[2,3] \times[0,1]} \\
5 . & {[2,3] \times[0,1] \times[1,3]} & {[2,3] \times[0,1] \times[1,3]} \\
6 . & {[2,3] \times[1,3] \times[1,2]} & {[2,3] \times[1,3] \times[1,2]} \\
7 . & {[3,4] \times[3,5] \times[5,6]} & {[3,4] \times[3,5] \times[4,5]} \\
8 . & {[3,4] \times[4,5] \times[3,5]} & {[3,4] \times[5,6] \times[3,5]} \\
9 . & {[3,5] \times[5,6] \times[3,4]} & {[3,5] \times[3,4] \times[5,6]} \\
10 . & {[3,5] \times[3,4] \times[4,5]} & {[3,5] \times[4,5] \times[3,4]} \\
11 . & {[4,5] \times[3,5] \times[3,4]} & {[4,5] \times[3,4] \times[3,5]} \\
12 . & {[5,6] \times[3,4] \times[3,5]} & {[5,6] \times[3,5] \times[3,4]}
\end{array}
$$

Table 4: A system of representatives for $\mathrm{Cq}^{\uparrow} / A^{3}(5)$.
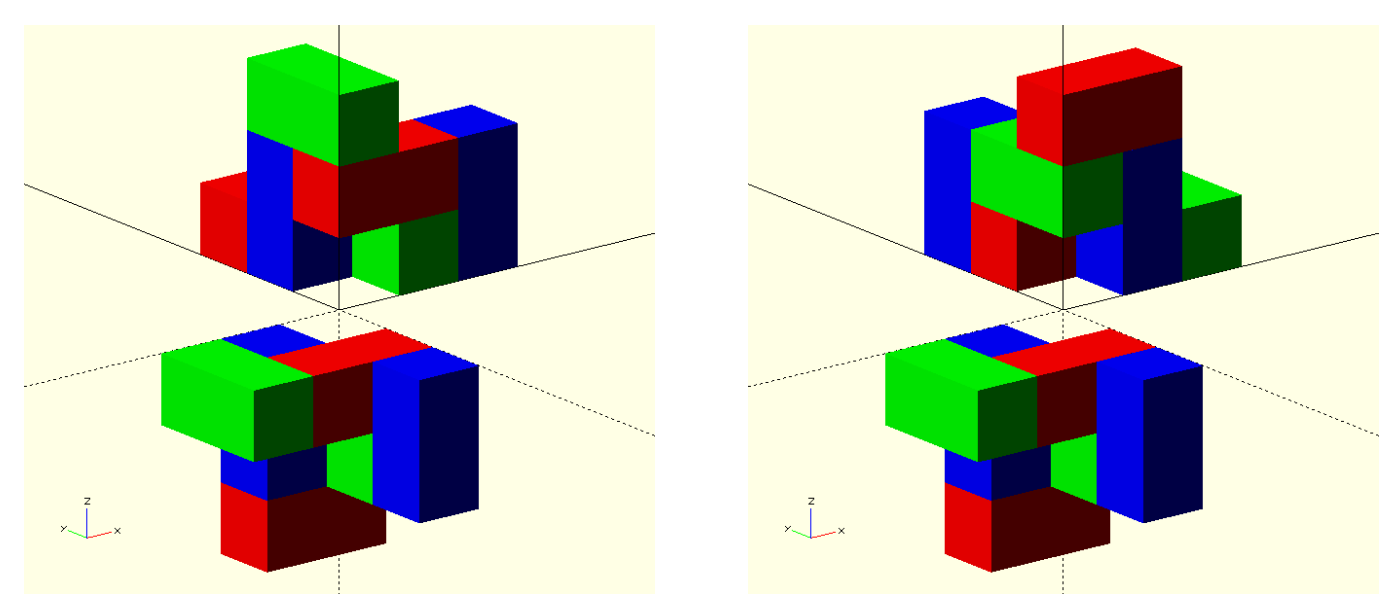

Figure 7: Illustrations of cliques $\mathscr{D}^{1}$ and $\mathscr{D}^{2}$. They are translated so that their circumscribed boxes are centred at the origin.

It seems to be a proper place to give a formal definition of an isomorphism of cliques. For every pair of $n$-intervals $I$ and $J$ we define a $0 / 1$ vector $\varepsilon=\varepsilon(I, J)$ in $\mathbb{R}^{n}$ as follows

$$
\varepsilon_{i}= \begin{cases}1, & \text { if } I_{i} \text { and } J_{i} \text { have exactly one point in common } \\ 0, & \text { otherwise. }\end{cases}
$$




$$
\left[\begin{array}{llllllllllll}
0 & 1 & 1 & 1 & 2 & 4 & 2 & 4 & 2 & 4 & 6 & 6 \\
1 & 0 & 2 & 4 & 1 & 1 & 2 & 4 & 2 & 4 & 6 & 6 \\
1 & 2 & 0 & 2 & 2 & 4 & 1 & 5 & 1 & 5 & 4 & 4 \\
1 & 4 & 2 & 0 & 4 & 4 & 3 & 1 & 3 & 1 & 2 & 2 \\
2 & 1 & 2 & 4 & 0 & 2 & 1 & 5 & 1 & 5 & 4 & 4 \\
4 & 1 & 4 & 4 & 2 & 0 & 3 & 1 & 3 & 1 & 2 & 2 \\
2 & 2 & 1 & 3 & 1 & 3 & 0 & 4 & 4 & 2 & 1 & 4 \\
4 & 4 & 5 & 1 & 5 & 1 & 4 & 0 & 2 & 2 & 1 & 2 \\
2 & 2 & 1 & 3 & 1 & 3 & 4 & 2 & 0 & 4 & 4 & 1 \\
4 & 4 & 5 & 1 & 5 & 1 & 2 & 2 & 4 & 0 & 2 & 1 \\
6 & 6 & 4 & 2 & 4 & 2 & 1 & 1 & 4 & 2 & 0 & 1 \\
6 & 6 & 4 & 2 & 4 & 2 & 4 & 2 & 1 & 1 & 1 & 0
\end{array}\right], \quad\left[\begin{array}{llllllllllll}
0 & 1 & 1 & 1 & 2 & 4 & 2 & 4 & 2 & 4 & 6 & 6 \\
1 & 0 & 2 & 4 & 1 & 1 & 2 & 4 & 2 & 4 & 6 & 6 \\
1 & 2 & 0 & 2 & 2 & 4 & 1 & 5 & 1 & 5 & 4 & 4 \\
1 & 4 & 2 & 0 & 4 & 4 & 3 & 1 & 3 & 1 & 2 & 2 \\
2 & 1 & 2 & 4 & 0 & 2 & 1 & 5 & 1 & 5 & 4 & 4 \\
4 & 1 & 4 & 4 & 2 & 0 & 3 & 1 & 3 & 1 & 2 & 2 \\
2 & 2 & 1 & 3 & 1 & 3 & 0 & 2 & 4 & 4 & 1 & 4 \\
4 & 4 & 5 & 1 & 5 & 1 & 2 & 0 & 4 & 2 & 1 & 2 \\
2 & 2 & 1 & 3 & 1 & 3 & 4 & 4 & 0 & 2 & 4 & 1 \\
4 & 4 & 5 & 1 & 5 & 1 & 4 & 2 & 2 & 0 & 2 & 1 \\
6 & 6 & 4 & 2 & 4 & 2 & 1 & 1 & 4 & 2 & 0 & 1 \\
6 & 6 & 4 & 2 & 4 & 2 & 4 & 2 & 1 & 1 & 1 & 0
\end{array}\right]
$$

Table 5: Adjacency matrices of $\mathscr{D}^{1}$ and $\mathscr{D}^{2}$.

Let $\mathscr{C}$ and $\mathscr{D}$ be two subfamilies of $\mathscr{I}^{n}$. A bijection $f: \mathscr{C} \rightarrow \mathscr{D}$ is an isomorphism between these families if there is a permutation $\sigma \in S_{n}$ so that

$$
s_{\sigma}(\varepsilon(f(I), f(J)))=\varepsilon\left(s_{\sigma}(f(I)), s_{\sigma}(f(J))\right)=\varepsilon(I, J)
$$

for every $I, J \in \mathscr{C}$. Obviously, if one of the two families is a clique, then the other is a clique as well. Isomorphic families will also be called combinatorially equivalent.

Observe that if $\mathscr{C} \in \mathrm{Cq}^{\boldsymbol{*}}$ and $\mathscr{D} \in \mathrm{Cq}^{\boldsymbol{\omega}}$, then they cannot be combinatorially equivalent; otherwise, $\mid E\left(\lambda^{\mathbf{*}}|=| E(\lambda) \mid\right.$, which is not true. Now, we are about to show that cliques $\mathscr{D}^{1}$ and $\mathscr{D}^{2}$ are not equivalent as well. Two approaches are effective:

(1) Prove that if cliques $\mathscr{C}$ and $\mathscr{D}$ belonging to $\mathrm{Cq}^{\oplus}$ are combinatorially equivalent, then there is $f \in A^{3}(5)$ such that $f(\mathscr{C})=\mathscr{D}$.

(2) Prove that authomorphism groups of $\mathscr{D}_{1}$ and $\mathscr{D}_{2}$ are not isomorphic.

We include both of them. Here we shall follow the second approach, while the first one is discussed in Appendix C.

For every finite family $\mathscr{D}=\left\{I^{1}, \ldots, I^{m}\right\} \subset \mathscr{I}^{n}$, we may define its adjacency matrix $A_{\mathscr{D}}=\left[\varepsilon_{i j}\right]$ so that $\varepsilon_{i j}=\varepsilon\left(I^{i}, I^{j}\right)$. Clearly, the automorphism group $\operatorname{Aut}(\mathscr{D})$ is isomorphic to the group of all these permutations $\pi \in S_{m}$ for whose there are $\sigma \in S_{n}$ such that $s_{\sigma}\left(\varepsilon_{\pi(i) \pi(j)}\right)=\varepsilon_{i j}$ for every $i$ and $j$. Table 5 contains the adjacency matrices of $\mathscr{D}^{1}$ and $\mathscr{D}^{2}$; in order to simplify the notation each $0 / 1$ wector $(\alpha, \beta, \gamma)$ is replaced by the number $\alpha+2 \beta+4 \gamma$.

The following simple idea can be applied in order to compute the automorphism group of $\mathscr{D}$ efficiently. Let $b_{i j}$ be the cardinality of the set $\left\{k: \varepsilon_{i k}=\varepsilon_{j k}\right\}$. Let $B_{\mathscr{D}}=\left[b_{i j}\right]$. A permutation $\pi \in S_{m}$ is a protoautomorphism of $\mathscr{D}$ if it fixes $B_{\mathscr{D}}$; that is, $\left[b_{i j}\right]=\left[b_{\pi(i) \pi(j)}\right]$. Clearly, each automorphism is a protoautomorphism. On the other hand, it is much easier 


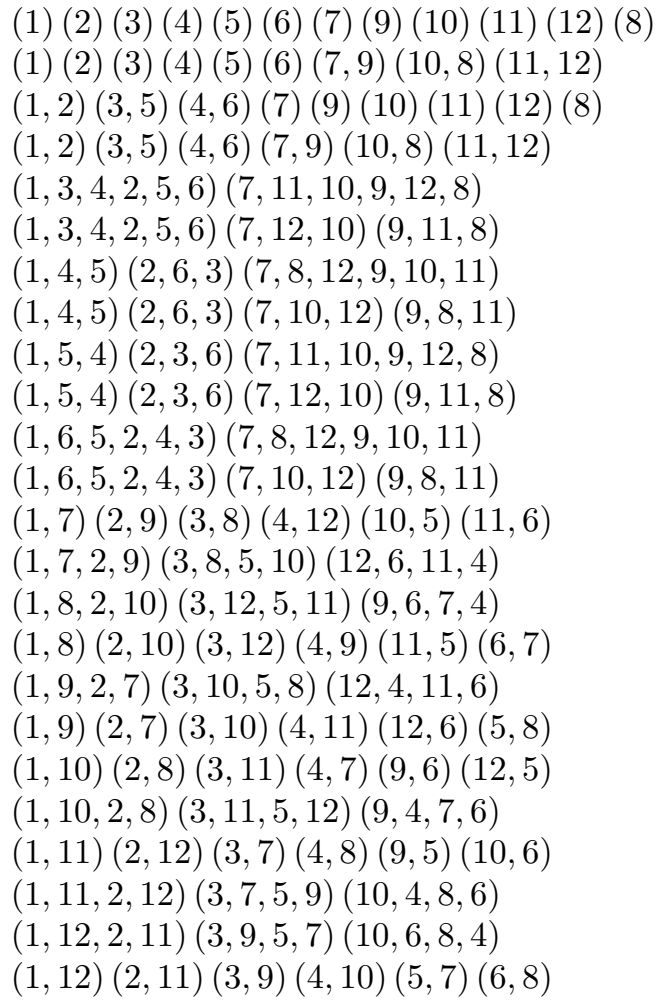

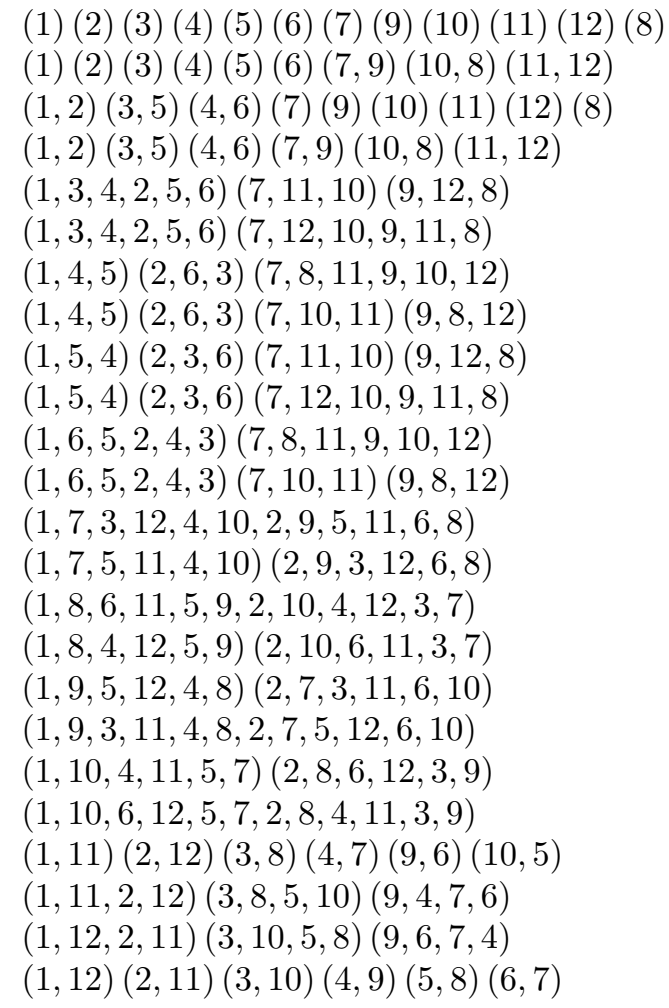

Table 6: Isomorphic copies of $\operatorname{Aut}\left(\mathscr{D}^{1}\right)$ and $\operatorname{Aut}\left(\mathscr{D}^{2}\right)$.

to determine whether a permutation is a protoautomorphism than an automorphism. It appears that both $\mathscr{D}^{1}$ and $\mathscr{D}^{2}$ have only 48 protoautomorphism. Finally, it remains to single out automorphisms from protoautomorphisms, which is an easy task as the number of protoautomorphisms is small. Again, it appears that both automorphism groups are of the same order 24. All automorphisms of both cliques are collected in Tabel 6. Clearly, groups $\operatorname{Aut}\left(\mathscr{D}^{1}\right)$ and $\operatorname{Aut}\left(\mathscr{D}^{2}\right)$ are different, as only the latter contains elements of order 12. In fact, we can easily identify these groups using GAP [21]. The first of them is an (external) semidirect product of cyclic group $\mathbb{Z}_{3}$ by dihedral group $\operatorname{Dih}_{4}$ of order 8 , where the action of the latter on the former is given by a homomorphism whose kernel is one of the Klein four-subgroups of the dihedral group. The second is the simple product of $\mathbb{Z}_{3}$ and $\mathrm{Dih}_{4}$.

Proposition 20. The quotient space $\mathrm{Cq} / \approx$, where $\approx$ is the combinatorial equivalence of cliques, consists of two classes

Since all cliques belonging to $\mathrm{Cq}^{\text {* }}$ are isomorphic, their automorphism groups are isomorphic as well. Therefore, it suffices to fix any $\mathscr{C} \in \mathrm{Cq}^{\boldsymbol{*}}$, and find $\operatorname{Aut}(\mathscr{C})$ in order to know the structure of the automorphism groups of the remaining cliques. We could take for example clique $\mathscr{C}$ described in Example 1. By much the same argument as in the case of $\mathscr{D}^{i}$ one finds that $\operatorname{Aut}(\mathscr{C})$ is of order 48. (Interestingly enough, the protoautomorphism group has in this case 3070 elements). The following permuta- 


$$
\left[\begin{array}{llllllllllll}
0 & 1 & 1 & 1 & 2 & 4 & 2 & 4 & 2 & 4 & 6 & 6 \\
1 & 0 & 2 & 4 & 1 & 1 & 2 & 4 & 2 & 4 & 6 & 6 \\
1 & 2 & 0 & 2 & 2 & 4 & 1 & 4 & 1 & 4 & 4 & 4 \\
1 & 4 & 2 & 0 & 4 & 4 & 2 & 1 & 2 & 1 & 2 & 2 \\
2 & 1 & 2 & 4 & 0 & 2 & 1 & 4 & 1 & 4 & 4 & 4 \\
4 & 1 & 4 & 4 & 2 & 0 & 2 & 1 & 2 & 1 & 2 & 2 \\
2 & 2 & 1 & 2 & 1 & 2 & 0 & 4 & 4 & 2 & 1 & 4 \\
4 & 4 & 4 & 1 & 4 & 1 & 4 & 0 & 2 & 2 & 1 & 2 \\
2 & 2 & 1 & 2 & 1 & 2 & 4 & 2 & 0 & 4 & 4 & 1 \\
4 & 4 & 4 & 1 & 4 & 1 & 2 & 2 & 4 & 0 & 2 & 1 \\
6 & 6 & 4 & 2 & 4 & 2 & 1 & 1 & 4 & 2 & 0 & 1 \\
6 & 6 & 4 & 2 & 4 & 2 & 4 & 2 & 1 & 1 & 1 & 0
\end{array}\right], \quad\left[\begin{array}{llllllllllll}
0 & 1 & 1 & 1 & 2 & 4 & 2 & 4 & 2 & 4 & 6 & 6 \\
1 & 0 & 2 & 4 & 1 & 1 & 2 & 4 & 2 & 4 & 6 & 6 \\
1 & 2 & 0 & 2 & 2 & 4 & 1 & 4 & 1 & 4 & 4 & 4 \\
1 & 4 & 2 & 0 & 4 & 4 & 2 & 1 & 2 & 1 & 2 & 2 \\
2 & 1 & 2 & 4 & 0 & 2 & 1 & 4 & 1 & 4 & 4 & 4 \\
4 & 1 & 4 & 4 & 2 & 0 & 2 & 1 & 2 & 1 & 2 & 2 \\
2 & 2 & 1 & 2 & 1 & 2 & 0 & 2 & 4 & 4 & 1 & 4 \\
4 & 4 & 4 & 1 & 4 & 1 & 2 & 0 & 4 & 2 & 1 & 2 \\
2 & 2 & 1 & 2 & 1 & 2 & 4 & 4 & 0 & 2 & 4 & 1 \\
4 & 4 & 4 & 1 & 4 & 1 & 4 & 2 & 2 & 0 & 2 & 1 \\
6 & 6 & 4 & 2 & 4 & 2 & 1 & 1 & 4 & 2 & 0 & 1 \\
6 & 6 & 4 & 2 & 4 & 2 & 4 & 2 & 1 & 1 & 1 & 0
\end{array}\right]
$$

Table 7: Adjacency matrices of $\mathscr{C}^{1}$ and $\mathscr{C}^{2}$.

tions are generators of the isomorphic copy of $\operatorname{Aut}(\mathscr{C}):(1,12,7,4,9,6)(2,10,5,3,11,8)$, $(1,12,4,9)(2,11,3,10)(5,6,8,7)$. Since $\left|A^{3}(s)\right|=\left|S_{3}\right||\operatorname{Aut}(s)|^{3}=6 \cdot 8^{3}$, for $s \geqslant 4$, $|\operatorname{Aut}(\mathscr{C})|=48$ and $\left|\mathrm{Cq}^{*}\right|=64$, it follows that each automorphism of $\mathscr{C}$ is the restriction to $\mathscr{C}$ of an element from $A^{3}(4)$. Similarly, all automorphisms of $\mathscr{D}^{1}$ and $\mathscr{D}^{2}$ can be identified with corresponding elements of $A^{3}(5)$.

\section{Compressible cliques.}

We have classified all incompressible 12-cliques in $\mathscr{I}^{3}$ up to combinatorial equivalence, however, it is not the whole picture. There are 12-cliques which are of different combinatorial type from the three described so far. Clearly, if $\mathscr{C}$ is such a clique, then there is a homomorphism $f=f_{1} \times f_{2} \times f_{2}: \mathscr{I}^{3} \rightarrow \mathscr{I}(s)^{3}$, where $s \in\{4,5\}$ such that $\mathscr{D}=f(\mathscr{C})$ is an incompressible clique (see Section 5). Since $\varepsilon(I, J) \leqslant \varepsilon(f(I), f(J))$, for every $I, J \in \mathscr{I}^{3}$, we deduce that $A_{\mathscr{C}} \leqslant A_{\mathscr{D}}$, where both inequalities are stated with respect to the coordinatewise order. Since we have only three combinatorial types of incompressible cliques, we deduce that the number of combinatorial types of 12-cliques is finite. As we briefly explain in this section, this number is 5 . Details will be published elsewhere.

It can be shown that if $A_{\mathscr{C}} \neq A_{\mathscr{D}}$, then $\mathscr{D} \in \mathrm{Cq}^{\star}$. As our objective is to characterize all 12-cliques up to combinatorial equivalence, we may assume that $\mathscr{D} \in\left\{\mathscr{D}^{1}, \mathscr{D}^{2}\right\}$. Let us assume that the intervals belonging to $\mathscr{D}=\mathscr{D}^{i}$ are labelled as in Table 4. Moreover, let the elements of $\mathscr{C}=\mathscr{C}^{i}$ be labelled so that $I=f(J) \in \mathscr{D}$ and $J \in \mathscr{C}$ have the same number. If we take into account authomorphisms of $\mathscr{D}$, the fact that $\varepsilon\left(I^{1}, I^{8}\right)=(1,0,1)$ and the assumption $A_{\mathscr{C}} \neq A_{\mathscr{D}}$, then we can set that $\varepsilon\left(J^{1}, J^{8}\right)=(0,0,1)$. It appears that this equality determines $A_{\mathscr{C}}$ for both $\mathscr{D}^{1}$ and $\mathscr{D}^{2}$. In Table 7 , the adjacency matrices of $\mathscr{C}^{1}$ and $\mathscr{C}^{2}$ encoded in the same manner as in Table 5 are presented. The subsequent table shows the corresponding 12-cliques. 


$\begin{array}{ccc}\text { 1. } & {[0,1] \times[1,3] \times[2,3]} & {[0,1] \times[1,3] \times[2,3]} \\ 2 . & {[1,2] \times[2,3] \times[1,3]} & {[1,2] \times[2,3] \times[1,3]} \\ 3 . & {\left[1, \frac{5}{2}\right] \times[1,2] \times[2,3]} & {\left[1, \frac{5}{2}\right] \times[1,2] \times[2,3]} \\ 4 . & {[1,3] \times[2,3] \times[0,1]} & {[1,3] \times[2,3] \times[0,1]} \\ 5 . & {\left[2, \frac{5}{2}\right] \times[0,1] \times[1,3]} & {\left[2, \frac{5}{2}\right] \times[0,1] \times[1,3]} \\ 6 . & {[2,3] \times[1,3] \times[1,2]} & {[2,3] \times[1,3] \times[1,2]} \\ \text { 7. } & {\left[\frac{5}{2}, 4\right] \times[3,5] \times[5,6]} & {\left[\frac{5}{2}, 4\right] \times[3,5] \times[4,5]} \\ 8 . & {[3,4] \times[4,5] \times[3,5]} & {[3,4] \times[5,6] \times[3,5]} \\ 9 . & {\left[\frac{5}{2}, 5\right] \times[5,6] \times[3,4]} & {\left[\frac{5}{2}, 5\right] \times[3,4] \times[5,6]} \\ 10 . & {[3,5] \times[3,4] \times[4,5]} & {[3,5] \times[4,5] \times[3,4]} \\ 11 . & {[4,5] \times[3,5] \times[3,4]} & {[4,5] \times[3,4] \times[3,5]} \\ 12 . & {[5,6] \times[3,4] \times[3,5]} & {[5,6] \times[3,5] \times[3,4]}\end{array}$

Table 8: Compressible cliques $\mathscr{C}^{1}, \mathscr{C}^{2}$.

It can be shown that $\mathscr{C}^{1}$ and $\mathscr{C}^{2}$ are not isomorphic, despite the fact that their automorphism groups are both isomorphic to $\mathrm{Dih}_{4}$.
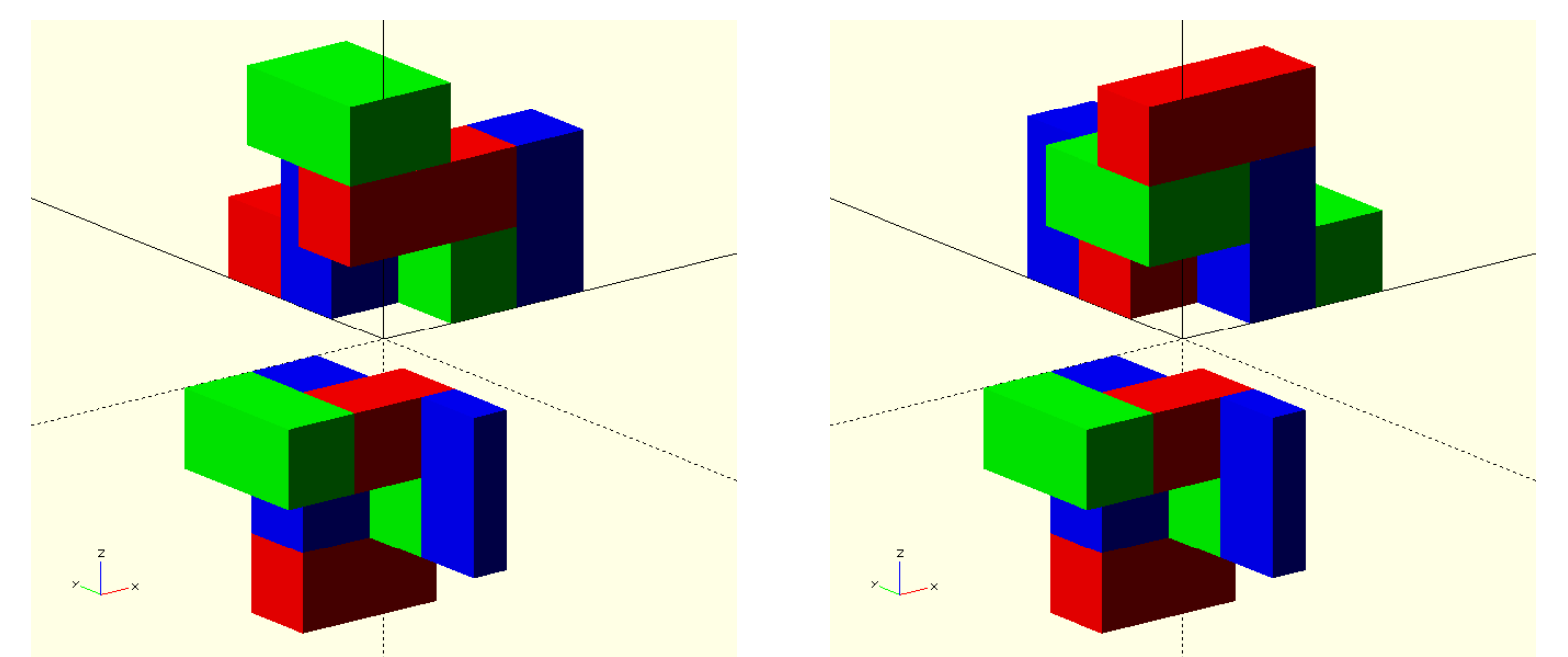

Figure 8: Illustrations of compressible cliques $\mathscr{C}^{1}$ and $\mathscr{C}^{2}$. They are translated so that their circumscribed boxes are centred at the origin.

\section{Isometric incompressible cliques. Chirality}

Let $I(s)=[0, s+1] \times[0, s+1] \times[0, s+1]$. Let $\operatorname{Iso}^{3}(s)$ be the group of isometries of the cube $I(s)$. For $s=4, \operatorname{Iso}^{3}(s)$ acts on $\mathrm{Cq}^{*}$, while for $s=5, \operatorname{Iso}^{3}(s)$ acts on $\mathrm{Cq}^{\star}$. The action is defined naturally: If $U \in \operatorname{Iso}^{3}(s)$ and $\mathscr{C}$ is an incompressible 12-clique, then $U(\mathscr{C})=\{U(I): I \in \mathscr{C}\}$. Let us remark, that $\operatorname{Iso}^{3}(s)$ can be identified with the subgroup of $A^{3}(s)$ consisting of all the elements $s_{\sigma} \circ f=s_{\sigma} \circ\left(f_{1} \times f_{2} \times f_{3}\right)$ such that $\sigma$ is an arbitrary 

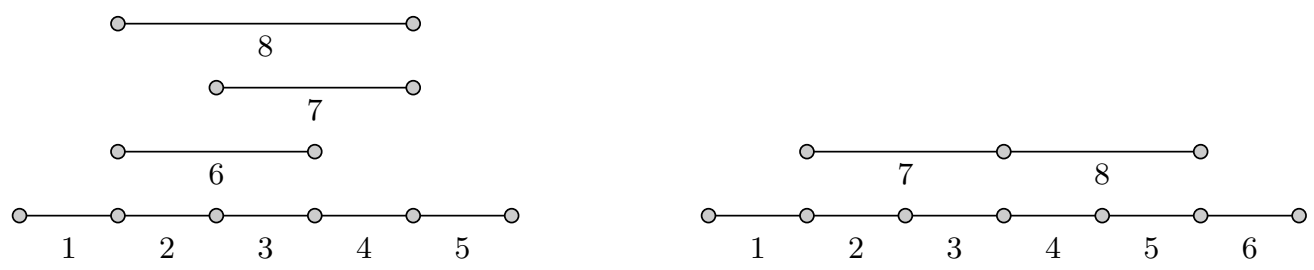

Figure 9: Labelings of supp $\lambda^{\boldsymbol{\bullet}}$ and $\operatorname{supp} \lambda^{\boldsymbol{\uparrow}}$.

permutation belonging to $S_{3}$ while each $f_{i}$ is either the identity mapping or the reflection about $(s+1) / 2$; that is, $f_{i}([a, b])=[s+1-b, s+1-a]$, for $[a, b] \in \mathscr{I}(s)$.

Each group $\operatorname{Iso}^{3}(s)$ contains the subgroup $\operatorname{Iso}_{+}^{3}(s)$ consisting of preserving-orientation isometries. Since incompressible cliques of intervals are geometric objects, it makes sense to characterize them up to congruency; that is, to find a system of representatives for

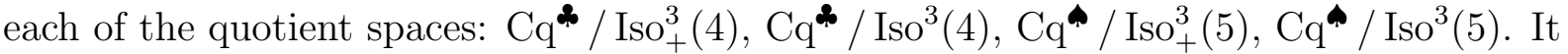
appears that some of the cliques are chiral while the other are achiral. In our context, a clique $\mathscr{C}$ is achiral if its orbits with respect to $\operatorname{Iso}_{+}^{3}(s)$ and $\operatorname{Iso}^{3}(s)$ coincide; otherwise, it is chiral.

To simplify the exposition, we label the elements of supp $\lambda^{\boldsymbol{n}}$ as follows: $[i-1, i] \mapsto i$, for $i=1, \ldots, 5 ;[1,3] \mapsto 6,[2,4] \mapsto 7,[1,4] \mapsto 8$. Then we extend this labelling to 3intervals; for example, interval $I=[0,1] \times[3,4] \times[1,4]$ is labeled by 148 . Similarly we label the elements of of supp $\lambda^{\curvearrowleft: ~}[i-1, i] \mapsto i$, for $i=1, \ldots, 6 ;[1,3] \mapsto 7,[3,5] \mapsto 8$ (Figure 8). We gather the information concerning the quotient spaces under the discussion in Tables $9-10$

\begin{tabular}{|ccc|}
\hline No. & orbit representative & orbit length \\
\hline 1. & {$[148,157,246,253,624,814,325,715,462,481,532,571]$} & 8 \\
2. & {$[165,134,285,274,651,852,341,742,413,427,516,528]$} & 8 \\
\hline 3. & {$[116,128,213,227,652,842,351,741,465,434,585,574]$} & 24 \\
4. & {$[116,123,218,227,645,855,344,754,482,471,562,531]$} & 24 \\
\hline
\end{tabular}

Table 9: A system of representatives for $\mathrm{Cq}^{\boldsymbol{\alpha}} / \mathrm{Iso}_{+}^{3}(4)$. Two blocks of cliques belonging to the same orbit of $\operatorname{Iso}^{3}(4)$ are distinguished. All cliques are chiral.

\section{Acknowledgements and concluding remarks.}

We extensively used solvers for mixed integer programming in the early stages of our investigations. Our first (incompressible) 12-clique of 3-intervals was found using A. Makhorin's GLPK (Gnu Linear Programming Kit) for Windows [12]. Other incompressible cliques were produced with the aid of SCIP Optimization Suite $[1,10]$. Gurobi Optimizer 5.1 was employed to perform preliminary verification that no additional incompressible cliques existed. Subsequently, we wrote Python scripts to make the results mathematically sound. Automorphism groups were calculated with the use of GAP[21]. 


\begin{tabular}{|ccc|}
\hline No. & orbit representative & orbit length \\
\hline I. & class of the combinatorial equivalence $\approx$ & \\
\hline 1. & {$[177,233,727,732,313,371,445,464,886,858,548,684]$} & 12 \\
2. & {$[177,233,723,772,317,331,446,454,885,868,584,648]$} & 12 \\
\hline 3. & {$[177,233,727,732,313,371,446,468,885,854,544,688]$} & 12 \\
4. & {$[177,233,723,772,317,331,486,464,845,858,544,688]$} & 12 \\
\hline 5. & {$[177,233,727,732,313,371,485,454,846,868,588,644]$} & 12 \\
6. & {$[177,233,723,772,317,331,445,458,886,864,588,644]$} & 12 \\
\hline 7. & {$[177,233,727,732,313,371,486,458,845,864,584,648]$} & 24 \\
8. & {$[177,233,723,772,317,331,485,468,846,854,548,684]$} & 24 \\
\hline 9. & {$[173,237,723,731,317,372,486,458,845,864,584,648]$} & 4 \\
10. & {$[137,273,713,732,327,371,485,468,846,854,548,684]$} & 4 \\
\hline II. & class of the combinatorial equivalence $\approx$ & \\
\hline 11. & {$[177,233,727,732,313,371,445,458,886,864,588,644]$} & 24 \\
12. & {$[177,233,727,732,313,371,446,454,885,868,584,648]$} & 24 \\
\hline 13. & {$[177,233,727,732,313,371,485,468,846,854,548,684]$} & 24 \\
14. $[177,233,723,772,317,331,486,458,845,864,584,648]$ & 24 \\
\hline 15. & {$[177,233,727,732,313,371,486,464,845,858,544,688]$} & 24 \\
\hline 16. & {$[173,237,723,731,317,372,485,468,846,854,548,684]$} & 8 \\
\hline
\end{tabular}

Table 10: A system of representatives for $\mathrm{Cq}^{\star} / \mathrm{Iso}_{+}^{3}(5)$. There are two blocks of cliques corresponding to two classes of the combinatorial equivalence. Each block consists of subblocks. Cliques within a subblock belong to the same orbit of $\mathrm{Iso}^{3}(5)$. Cliques 15 and 16 are achiral.

We wish to thank Professor J. Zaks for sending us reprints of his papers.

We are greatly indebted to an anonymous reviewer for valuable comments and suggestions. The alternative approach to the classification of maximum cliques presented in Appendix C has been proposed by the reviewer.

\section{Appendices}

\section{A $\operatorname{Aut}(s)$}

Our goal is to describe the group $\operatorname{Aut}(s)$ of all automorphisms of the graph $\mathscr{I}(s)$ (see Section 3 for the definition of $\mathscr{I}(s)$ ). If $s \in\{1,2\}$, then $\mathscr{I}(s)$ is a path of length $s$ and consequently $\operatorname{Aut}(s)$ is isomorphic to $\mathbb{Z}_{2}$. If $s=3$, then $\mathscr{I}(s)$ is a cycle of length 5. Therefore, Aut(3) is isomorphic to $\mathrm{Dih}_{5}$, which can be identified with the group of isometries of a regular pentagon. In the case of $s=4$, one can see that $\operatorname{Aut}(s)$ is isomorphic to $\mathrm{Dih}_{4}$, the group of isometries of a square. Figure 10 provides a sufficient explanation. 

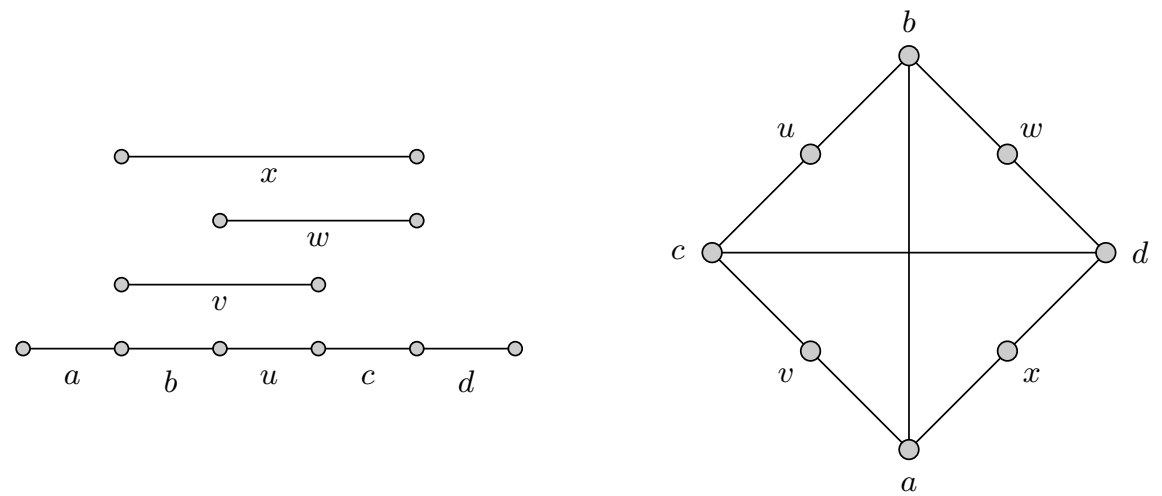

Figure 10: Diagrams for $\mathscr{I}(4)$.

In fact, the latter statement remains valid for arbitrary $s \geqslant 4$ :

Theorem 21. Aut $(s) \cong \operatorname{Dih}_{4}$, for every $s \geqslant 4$.

We are going to prove this result straightforwardly; that is, by determining all the elements of $\operatorname{Aut}(s)$. We begin with analysing the degrees of the vertices of $\mathscr{I}(s)$. We adopt the following shorthand notation: Each vertex (interval) $[i, j]$ of the graph $\mathscr{I}(s)$ is denoted by $i: j$.

Lemma 22. Let $s \geqslant 4$. Let $i: j \in V(\mathscr{I}(s))$. Then $\operatorname{deg}(i: j)$ is equal to:

1. $s-(j-i)-1$, if $2 \leqslant i$ and $j \leqslant s-1$;

2. $s-(j-i)$, if $i, j$ satisfy one of the following relations: $i=0$ and $j=1, i=1$ and $j \leqslant s-1,2 \leqslant i$ and $j=s, i=s$ and $j=s+1$;

3. $s-(j-i)+1=2$, if $i=1$ and $j=s$.

The proof is obvious, the degree $\operatorname{deg}(i: j)$ is equal to the number of the intervals in $\mathscr{I}(s)$ adjacent to $i: j$. For example, if $i=1$ and $j \leqslant s-1$, then the only interval adjacent to $i: j$ from the left is $0: 1$, while the intervals $j: k$, where $j+1 \leqslant k \leqslant s$, are adjacent from the right. Therefore, there are $1+(s-j)=s-(j-i)$ adjacent intervals in total, a result which agrees with our lemma.

Proof of the theorem. It follows from the lemma that if $s \geqslant 4$, then the only vertices of degree $s-1$ in $\mathscr{I}(s)$ are $a=0: 1, b=1: 2, c=s-1: s$ and $d=s: s+1$. Thus, every automorphism of $\mathscr{I}(s)$ has to send these vertices onto themselves. Since the subgraph $G$ of $\mathscr{I}(s)$ induced by $V=\{a, b, c, d\}$ is a disjoint union of two paths of length 1 , its automorphism group $\operatorname{Aut}(G)$ is isomorphic to $\operatorname{Dih}_{4}$. We shall prove that every automorphism of $G$ extends to an automorphism of $\mathscr{I}(s)$ in a unique way. Let us begin with listing the 
automorphisms of $G$.

\begin{tabular}{|l||l|l|l|l|}
\hline 1 & $a$ & $b$ & $c$ & $d$ \\
\hline 2 & $d$ & $c$ & $a$ & $b$ \\
\hline 3 & $b$ & $a$ & $d$ & $c$ \\
\hline 4 & $c$ & $d$ & $b$ & $a$ \\
\hline 5 & $b$ & $a$ & $c$ & $d$ \\
\hline 6 & $a$ & $b$ & $d$ & $c$ \\
\hline 7 & $d$ & $c$ & $b$ & $a$ \\
\hline 8 & $c$ & $d$ & $a$ & $b$ \\
\hline
\end{tabular}

\begin{tabular}{|c||c|c|c|c|}
\hline 1 & $0: 1$ & $1: 2$ & $s-1: s$ & $s: s+1$ \\
\hline 2 & $s: s+1$ & $s-1: s$ & $0: 1$ & $1: 2$ \\
\hline 3 & $1: 2$ & $0: 1$ & $s: s+1$ & $s-1: s$ \\
\hline 4 & $s-1: s$ & $s: s+1$ & $1: 2$ & $0: 1$ \\
\hline 5 & $1: 2$ & $0: 1$ & $s-1: s$ & $s: s+1$ \\
\hline 6 & $0: 1$ & $1: 2$ & $s: s+1$ & $s-1: s$ \\
\hline 7 & $s: s+1$ & $s-1: s$ & $1: 2$ & $0: 1$ \\
\hline 8 & $s-1: s$ & $s: s+1$ & $0: 1$ & $1: 2$ \\
\hline
\end{tabular}

Lists of the automorphisms of $G$ in both notations for vertices.

These lists encode automorphisms in a rather obvious manner; for example, the automorphism defined by the row no. 5 acts as follows: $a \mapsto b, b \mapsto a, c \mapsto c, d \mapsto d$.

Let us consider the vertices of the lowest degree in $\mathscr{I}(s), s \geqslant 4$. These are $u=2: s-1$, $v=1: s-1, w=2: s$ oraz $x=1: s$. The graph $H$ induced by the vertices $a, b, c, d, u, v$, $w, x$ is isomorphic to $\mathscr{I}(4)$ (for $s=4$ they simply coincide) and Figure 10 represents $H$ equally well as it represents $\mathscr{I}(4)$.

Clearly, the automorphisms of $G$ extend to the automorphisms of $H$ in a natural and unique way. We arrange the automorphisms of $H$ into two tables. The second table describes the action of these automorphisms restricted to the vertices $u, v, w, x$ in the original notation.

\begin{tabular}{|c||c|c|c|c|c|c|c|c|}
\hline 1 & $a$ & $b$ & $c$ & $d$ & $u$ & $v$ & $w$ & $x$ \\
\hline 2 & $d$ & $c$ & $a$ & $b$ & $v$ & $x$ & $u$ & $w$ \\
\hline 3 & $b$ & $a$ & $d$ & $c$ & $x$ & $w$ & $v$ & $u$ \\
\hline 4 & $c$ & $d$ & $b$ & $a$ & $w$ & $u$ & $x$ & $v$ \\
\hline 5 & $b$ & $a$ & $c$ & $d$ & $v$ & $u$ & $x$ & $w$ \\
\hline 6 & $a$ & $b$ & $d$ & $c$ & $w$ & $x$ & $u$ & $v$ \\
\hline 7 & $d$ & $c$ & $b$ & $a$ & $u$ & $w$ & $v$ & $x$ \\
\hline 8 & $c$ & $d$ & $a$ & $b$ & $x$ & $v$ & $w$ & $u$ \\
\hline
\end{tabular}

\begin{tabular}{|l||l|l|l|l|}
\hline 1 & $2: s-1$ & $1: s-1$ & $2: s$ & $1: s$ \\
\hline 2 & $1: s-1$ & $1: s$ & $2: s-1$ & $2: s$ \\
\hline 3 & $1: s$ & $2: s$ & $1: s-1$ & $2: s-1$ \\
\hline 4 & $2: s$ & $2: s-1$ & $1: s$ & $1: s-1$ \\
\hline 5 & $1: s-1$ & $2: s-1$ & $1: s$ & $2: s$ \\
\hline 6 & $2: s$ & $1: s$ & $2: s-1$ & $1: s-1$ \\
\hline 7 & $2: s-1$ & $2: s$ & $1: s-1$ & $1: s$ \\
\hline 8 & $1: s$ & $1: s-1$ & $2: s$ & $2: s-1$ \\
\hline
\end{tabular}

$\operatorname{Aut}(H)$

Now, we are prepared to describe all the automorphisms of $\mathscr{I}(s), s \geqslant 4$. Since, as we shall show, they are uniquely determined by the automorphisms of $G$, it suffices to describe their action on vertices belonging to $\mathscr{I}(s) \backslash V(H)$. These are the vertices of degree greater than 2 and smaller than $s-1$. The indices $i, j$ appearing in our description have to satisfy the following constraints:

$$
2<i<s-1 ; \quad i<j \text {, if both indices appear; } \quad 2<j<s-1 .
$$


The enumeration of automorphisms is consistent with the preceding tables.

\begin{tabular}{|c||c|c|l|l|c|}
\hline 1 & $1: i$ & $2: i$ & $j: s-1$ & $j: s$ & $i: j$ \\
\hline 2 & $s+1-i: s$ & $s+1-i: s-1$ & $1: s+1-j$ & $2: s+1-j$ & $s+1-j: s+1-i$ \\
\hline 3 & $2: i$ & $1: i$ & $j: s$ & $j: s-1$ & $i: j$ \\
\hline 4 & $s+1-i: s-1$ & $s+1-i: s$ & $2: s+1-j$ & $1: s+1-j$ & $s+1-j: s+1-i$ \\
\hline 5 & $2: i$ & $1: i$ & $j: s-1$ & $j: s$ & $i: j$ \\
\hline 6 & $1: i$ & $2: i$ & $j: s$ & $j: s-1$ & $i: j$ \\
\hline 7 & $s+1-i: s$ & $s+1-i: s-1$ & $2: s+1-j$ & $1: s+1-j$ & $s+1-j: s+1-i$ \\
\hline 8 & $s+1-i: s-1$ & $s+1-i: s$ & $1: s+1-j$ & $2: s+1-j$ & $s+1-j: s+1-i$ \\
\hline
\end{tabular}

The action of $\operatorname{Aut}(s)$ on $\mathscr{I}(s) \backslash V(H)$.

The fact that the described eight mappings, call them $\varphi_{i}, i=1, \ldots, 8$, are automorphisms of the graph $\mathscr{I}(s), s \geqslant 4$, is easily verified. As they extend the automorphism of $G$, it remains to be shown that no other extensions exist. Let $\varphi$ be any of the automorphisms. Then there is a unique $i$, such that $\varphi\left|V(G)=\varphi_{i}\right| V(G)$. As we have already mentioned, the extension of $\varphi_{i} \mid V(G)$ to $V(H)$ is unique; therefore, $\varphi\left|V(H)=\varphi_{i}\right| V(H)$. Since $H=\mathscr{I}(s)$ for $s=4$, we may further assume $s \geqslant 5$. Observe now, that for every $y \in V(G)$, and $2<\delta<s-1$ there is exactly one neighbouring vertex $z$ of degree $\delta$. Let $y^{\prime}=\varphi_{i}(y)=\varphi(y)$. Since $y^{\prime} \in V(G)$, there is only one vertex $z^{\prime}$ being a neighbour of $y^{\prime}$ whose degree is $\delta$. This implies that $z^{\prime}=\varphi(z)=\varphi_{i}(z)$. Thus, $\varphi$ and $\varphi_{i}$ coincide on the neighbourhood of $V(G)$. Now, if a vertex $t$ does not belong to the neighbourhood of $V(G)$, then there are $i, j$ such that $t=i: j$ and $2<i<j<s-1$. Take $z_{1}=1: i$ oraz $z_{2}=j: s$. These are neighbours of $t$. Moreover, they belong to the neighbourhood of $V(G)$, as the pairs $z_{1}, a$ and $z_{2}, d$ are adjacent. As a result, both automorphisms send $z_{1}$ on the same element $z_{1}^{\prime}$ and $z_{2}$ onto $z_{2}^{\prime}$. Since $z_{1}, z_{2}$ have a unique common neighbour $t$, the elements $z_{1}^{\prime}, z_{2}^{\prime}$ have a unique common member $t^{\prime}$. Clearly, $t^{\prime}=\varphi(t)=\varphi_{i}(t)$, which completes the proof that $\varphi$ and $\varphi_{i}$ coincide.

\section{B $\hat{L}(s, v)$}

We fulfill our declaration made in Section 5 to collect all $\hat{L}(s, v)$ for $v=12,13$. We explain how to read these tables taking row no. 9 of Table 13 as an example: Entries of this row bounded by double lines encode $\gamma=\lambda^{*}$, the combination discussed in Proposition 15 . The fact that $e=22^{\prime}$ means that the graph $G_{\gamma}$ has 22 edges and is of type II.

\begin{tabular}{|c||c|c|c|c|c||c|}
\hline No. & $0: 1$ & $1: 2$ & $1: 3$ & $2: 3$ & $3: 4$ & $e$ \\
\hline 1 & 2 & 2 & 2 & 3 & 3 & 29 \\
\hline
\end{tabular}

Table 11: $\hat{L}(s, v)$ for $v=12$ and $s=3$. 


\begin{tabular}{|c||c|c|c|c|c|c|c|c|c|}
\hline No. & $0: 1$ & $1: 2$ & $1: 3$ & $1: 4$ & $2: 3$ & $2: 4$ & $3: 4$ & $4: 5$ & $e$ \\
\hline 1 & 1 & 1 & 1 & 1 & 1 & 2 & 2 & 3 & 25 \\
\hline 2 & 1 & 1 & 1 & 1 & 1 & 1 & 3 & 3 & 26 \\
\hline 3 & 1 & 2 & 0 & 1 & 1 & 3 & 1 & 3 & 27 \\
\hline 4 & 1 & 2 & 1 & 1 & 1 & 2 & 2 & 2 & 24 \\
\hline 5 & 1 & 2 & 0 & 1 & 2 & 2 & 2 & 2 & 25 \\
\hline 6 & 1 & 2 & 0 & 1 & 1 & 2 & 2 & 3 & 26 \\
\hline 7 & 1 & 2 & 0 & 2 & 1 & 2 & 1 & 3 & 26 \\
\hline 8 & 2 & 2 & 1 & 1 & 1 & 1 & 2 & 2 & 24 \\
\hline
\end{tabular}

Table 12: $\hat{L}(s, v)$ for $v=12$ and $s=4$. 


\begin{tabular}{|c||c|c|c|c|c|c|c|c|c|c|c|c||c|}
\hline No. & $0: 1$ & $1: 2$ & $1: 3$ & $1: 4$ & $1: 5$ & $2: 3$ & $2: 4$ & $2: 5$ & $3: 4$ & $3: 5$ & $4: 5$ & $5: 6$ & $e$ \\
\hline 1 & 1 & 1 & 1 & 0 & 1 & 2 & 1 & 0 & 1 & 2 & 1 & 1 & 21 \\
\hline 2 & 1 & 1 & 1 & 0 & 1 & 2 & 1 & 0 & 2 & 1 & 1 & 1 & 21 \\
\hline 3 & 1 & 1 & 1 & 0 & 0 & 3 & 0 & 0 & 1 & 3 & 1 & 1 & 26 \\
\hline 4 & 1 & 1 & 1 & 0 & 0 & 3 & 0 & 0 & 2 & 2 & 1 & 1 & 26 \\
\hline 5 & 1 & 1 & 1 & 0 & 1 & 1 & 2 & 0 & 1 & 1 & 2 & 1 & 20 \\
\hline 6 & 1 & 1 & 1 & 0 & 1 & 1 & 1 & 1 & 1 & 1 & 1 & 2 & 20 \\
\hline 7 & 1 & 1 & 1 & 0 & 1 & 1 & 0 & 1 & 2 & 1 & 1 & 2 & 21 \\
\hline 8 & 1 & 1 & 1 & 0 & 1 & 1 & 1 & 0 & 1 & 2 & 1 & 2 & 21 \\
\hline 9 & 1 & 1 & 1 & 0 & 1 & 1 & 0 & 1 & 1 & 2 & 1 & 2 & 22 \\
\hline 10 & 1 & 1 & 1 & 0 & 0 & 2 & 0 & 1 & 2 & 1 & 1 & 2 & 22 \\
\hline 11 & 1 & 1 & 1 & 0 & 1 & 2 & 0 & 0 & 2 & 1 & 1 & 2 & 22 \\
\hline 12 & 1 & 1 & 1 & 0 & 0 & 2 & 0 & 1 & 1 & 2 & 1 & 2 & 23 \\
\hline 13 & 1 & 1 & 1 & 0 & 1 & 2 & 0 & 0 & 1 & 2 & 1 & 2 & 23 \\
\hline 14 & 1 & 1 & 1 & 0 & 0 & 2 & 0 & 0 & 2 & 2 & 1 & 2 & 24 \\
\hline 15 & 1 & 1 & 1 & 0 & 0 & 2 & 0 & 0 & 1 & 3 & 1 & 2 & 25 \\
\hline 16 & 1 & 1 & 1 & 0 & 0 & 1 & 0 & 2 & 1 & 1 & 1 & 3 & 22 \\
\hline 17 & 1 & 1 & 1 & 0 & 1 & 1 & 0 & 1 & 1 & 1 & 1 & 3 & 22 \\
\hline 18 & 1 & 1 & 1 & 0 & 0 & 1 & 0 & 1 & 1 & 2 & 1 & 3 & 23 \\
\hline 19 & 1 & 1 & 1 & 0 & 0 & 1 & 0 & 0 & 1 & 3 & 1 & 3 & 24 \\
\hline 20 & 1 & 1 & 1 & 0 & 0 & 1 & 1 & 1 & 1 & 1 & 2 & 2 & 21 \\
\hline 21 & 1 & 1 & 1 & 0 & 1 & 1 & 1 & 0 & 1 & 1 & 2 & 2 & 21 \\
\hline 22 & 1 & 1 & 1 & 0 & 0 & 1 & 0 & 1 & 2 & 1 & 2 & 2 & 22 \\
\hline 23 & 1 & 1 & 1 & 0 & 0 & 1 & 0 & 0 & 2 & 2 & 2 & 2 & 23 \\
\hline 24 & 1 & 1 & 1 & 0 & 0 & 2 & 0 & 0 & 1 & 2 & 2 & 2 & 23 \\
\hline 25 & 1 & 1 & 1 & 0 & 0 & 1 & 0 & 1 & 1 & 1 & 2 & 3 & 22 \\
\hline 26 & 1 & 1 & 1 & 0 & 0 & 1 & 0 & 0 & 1 & 2 & 2 & 3 & 23 \\
\hline 27 & 1 & 1 & 1 & 0 & 0 & 1 & 0 & 0 & 1 & 1 & 3 & 3 & 22 \\
\hline 28 & 1 & 1 & 2 & 0 & 0 & 2 & 0 & 0 & 2 & 2 & 1 & 1 & 26 \\
\hline 29 & 1 & 2 & 1 & 0 & 0 & 1 & 0 & 2 & 1 & 1 & 1 & 2 & 22 \\
\hline 30 & 1 & 2 & 1 & 0 & 0 & 1 & 1 & 1 & 1 & 1 & 1 & 2 & 21 \\
\hline 31 & 1 & 2 & 1 & 0 & 1 & 1 & 1 & 0 & 1 & 1 & 1 & 2 & 20 \\
\hline 32 & 1 & 2 & 1 & 0 & 0 & 1 & 0 & 1 & 1 & 2 & 1 & 2 & 22 \\
\hline 33 & 1 & 2 & 1 & 0 & 0 & 1 & 0 & 1 & 2 & 1 & 2 & 1 & 21 \\
\hline 34 & 1 & 2 & 1 & 0 & 0 & 2 & 0 & 0 & 1 & 2 & 1 & 2 & 23 \\
\hline 35 & 1 & 2 & 1 & 0 & 0 & 1 & 0 & 1 & 1 & 1 & 2 & 2 & 21 \\
\hline 36 & 1 & 2 & 1 & 0 & 0 & 2 & 0 & 0 & 1 & 1 & 2 & 2 & 21 \\
\hline 37 & 2 & 2 & 1 & 0 & 0 & 1 & 0 & 0 & 1 & 1 & 2 & 2 & 20 \\
\hline
\end{tabular}

Table 13: $\hat{L}(s, v)$ for $v=12$ and $s=5$. 


\begin{tabular}{|c||c|c|c|c|c|c|c|c|c|c|c|c|c|c|c|c|c||c|}
\hline No. & $0: 1$ & $1: 2$ & $1: 3$ & $1: 4$ & $1: 5$ & $1: 6$ & $2: 3$ & $2: 4$ & $2: 5$ & $2: 6$ & $3: 4$ & $3: 5$ & $3: 6$ & $4: 5$ & $4: 6$ & $5: 6$ & $6: 7$ & $e$ \\
\hline 1 & 1 & 1 & 1 & 0 & 0 & 0 & 1 & 1 & 0 & 0 & 1 & 1 & 1 & 1 & 1 & 1 & 1 & $19^{\prime}$ \\
\hline 2 & 1 & 1 & 1 & 0 & 0 & 0 & 1 & 1 & 0 & 1 & 1 & 1 & 0 & 1 & 1 & 1 & 1 & 18 \\
\hline 3 & 1 & 1 & 1 & 0 & 0 & 1 & 1 & 1 & 0 & 0 & 1 & 1 & 0 & 1 & 1 & 1 & 1 & 18 \\
\hline 4 & 1 & 1 & 1 & 0 & 0 & 0 & 1 & 0 & 0 & 0 & 2 & 1 & 1 & 1 & 1 & 1 & 1 & $20^{\prime}$ \\
\hline 5 & 1 & 1 & 1 & 0 & 0 & 0 & 1 & 0 & 0 & 1 & 2 & 1 & 0 & 1 & 1 & 1 & 1 & 19 \\
\hline 6 & 1 & 1 & 1 & 0 & 0 & 0 & 1 & 2 & 0 & 0 & 1 & 0 & 0 & 1 & 2 & 1 & 1 & 20 \\
\hline 7 & 1 & 1 & 1 & 0 & 0 & 0 & 1 & 1 & 0 & 0 & 1 & 0 & 1 & 1 & 2 & 1 & 1 & 19 \\
\hline 8 & 1 & 1 & 1 & 0 & 0 & 0 & 2 & 0 & 0 & 0 & 1 & 1 & 1 & 1 & 1 & 1 & 1 & $20^{\prime}$ \\
\hline 9 & 1 & 1 & 1 & 0 & 0 & 0 & 1 & 1 & 0 & 0 & 1 & 0 & 1 & 2 & 1 & 1 & 1 & 19 \\
\hline 10 & 1 & 1 & 1 & 0 & 0 & 0 & 1 & 1 & 0 & 0 & 2 & 0 & 0 & 1 & 2 & 1 & 1 & 21 \\
\hline 11 & 1 & 1 & 1 & 0 & 0 & 0 & 1 & 0 & 0 & 0 & 2 & 0 & 1 & 1 & 2 & 1 & 1 & 20 \\
\hline 12 & 1 & 1 & 1 & 0 & 0 & 0 & 1 & 0 & 0 & 0 & 2 & 0 & 1 & 2 & 1 & 1 & 1 & 20 \\
\hline 13 & 1 & 1 & 1 & 0 & 0 & 0 & 1 & 0 & 0 & 0 & 3 & 0 & 0 & 1 & 2 & 1 & 1 & 22 \\
\hline 14 & 1 & 1 & 1 & 0 & 0 & 0 & 1 & 0 & 0 & 0 & 1 & 0 & 2 & 1 & 1 & 1 & 2 & 20 \\
\hline 15 & 1 & 1 & 1 & 0 & 0 & 0 & 1 & 1 & 0 & 0 & 1 & 0 & 1 & 1 & 1 & 1 & 2 & 19 \\
\hline 16 & 1 & 1 & 1 & 0 & 0 & 0 & 1 & 0 & 0 & 1 & 1 & 0 & 1 & 1 & 1 & 1 & 2 & 19 \\
\hline 17 & 1 & 1 & 1 & 0 & 0 & 0 & 1 & 1 & 0 & 1 & 1 & 0 & 0 & 1 & 1 & 1 & 2 & 18 \\
\hline 18 & 1 & 1 & 1 & 0 & 0 & 0 & 1 & 0 & 0 & 0 & 1 & 1 & 1 & 1 & 1 & 1 & 2 & $19^{\prime}$ \\
\hline 19 & 1 & 1 & 1 & 0 & 0 & 0 & 1 & 0 & 0 & 1 & 1 & 1 & 0 & 1 & 1 & 1 & 2 & 18 \\
\hline 20 & 1 & 1 & 1 & 0 & 0 & 1 & 1 & 1 & 0 & 0 & 1 & 0 & 0 & 1 & 1 & 1 & 2 & 18 \\
\hline 21 & 1 & 1 & 1 & 0 & 0 & 0 & 1 & 0 & 0 & 0 & 2 & 0 & 1 & 1 & 1 & 1 & 2 & 20 \\
\hline 22 & 1 & 1 & 1 & 0 & 0 & 0 & 1 & 0 & 0 & 1 & 2 & 0 & 0 & 1 & 1 & 1 & 2 & 19 \\
\hline 23 & 1 & 1 & 1 & 0 & 0 & 0 & 1 & 0 & 0 & 0 & 1 & 0 & 1 & 1 & 2 & 1 & 2 & 19 \\
\hline 24 & 1 & 1 & 1 & 0 & 0 & 0 & 1 & 1 & 0 & 0 & 1 & 0 & 0 & 1 & 2 & 1 & 2 & 19 \\
\hline 25 & 1 & 1 & 1 & 0 & 0 & 0 & 1 & 0 & 0 & 0 & 1 & 0 & 1 & 2 & 1 & 2 & 1 & 18 \\
\hline 26 & 1 & 1 & 1 & 0 & 0 & 0 & 1 & 0 & 0 & 0 & 2 & 0 & 0 & 1 & 2 & 1 & 2 & 20 \\
\hline 27 & 1 & 1 & 1 & 0 & 0 & 0 & 1 & 0 & 0 & 0 & 1 & 0 & 1 & 1 & 1 & 2 & 2 & 19 \\
\hline 28 & 1 & 1 & 1 & 0 & 0 & 0 & 1 & 1 & 0 & 0 & 1 & 0 & 0 & 1 & 1 & 2 & 2 & 18 \\
\hline 29 & 1 & 1 & 1 & 0 & 0 & 0 & 1 & 0 & 0 & 0 & 2 & 0 & 0 & 1 & 1 & 2 & 2 & 19 \\
\hline
\end{tabular}

Table 14: $\hat{L}(s, v)$ for $v=12$ and $s=6$. 


\begin{tabular}{|c||c|c|c|c|c|c|c|c|c|c|c|c|c|c|c|c|c||c|}
\hline No. & $0: 1$ & $1: 2$ & $1: 3$ & $2: 3$ & $2: 4$ & $2: 5$ & $2: 7$ & $3: 4$ & $3: 5$ & $3: 7$ & $4: 5$ & $4: 6$ & $4: 7$ & $5: 6$ & $5: 7$ & $6: 7$ & $7: 8$ & $e$ \\
\hline 1 & 1 & 1 & 1 & 1 & 1 & 0 & 0 & 1 & 0 & 0 & 1 & 0 & 1 & 1 & 1 & 1 & 1 & 16 \\
\hline 2 & 1 & 1 & 1 & 1 & 0 & 0 & 0 & 1 & 1 & 0 & 1 & 0 & 1 & 1 & 1 & 1 & 1 & $17^{\prime}$ \\
\hline 3 & 1 & 1 & 1 & 1 & 0 & 0 & 1 & 1 & 1 & 0 & 1 & 0 & 0 & 1 & 1 & 1 & 1 & $17^{\prime}$ \\
\hline 4 & 1 & 1 & 1 & 1 & 0 & 0 & 0 & 1 & 2 & 0 & 1 & 0 & 0 & 1 & 1 & 1 & 1 & $19^{\prime}$ \\
\hline 5 & 1 & 1 & 1 & 1 & 0 & 0 & 0 & 1 & 0 & 1 & 1 & 0 & 1 & 1 & 1 & 1 & 1 & 16 \\
\hline 6 & 1 & 1 & 1 & 1 & 0 & 0 & 0 & 1 & 1 & 1 & 1 & 0 & 0 & 1 & 1 & 1 & 1 & $18^{\prime}$ \\
\hline 7 & 1 & 1 & 1 & 1 & 0 & 0 & 0 & 1 & 0 & 1 & 1 & 1 & 0 & 1 & 1 & 1 & 1 & 16 \\
\hline 8 & 1 & 1 & 1 & 1 & 0 & 1 & 0 & 1 & 0 & 1 & 1 & 0 & 0 & 1 & 1 & 1 & 1 & 17 \\
\hline 9 & 1 & 1 & 1 & 1 & 0 & 0 & 1 & 1 & 0 & 0 & 1 & 0 & 0 & 1 & 1 & 1 & 2 & 16 \\
\hline 10 & 1 & 1 & 1 & 1 & 0 & 0 & 0 & 1 & 1 & 0 & 1 & 0 & 0 & 1 & 1 & 1 & 2 & $17^{\prime}$ \\
\hline 11 & 1 & 1 & 1 & 1 & 0 & 0 & 0 & 1 & 0 & 1 & 1 & 0 & 0 & 1 & 1 & 1 & 2 & 17 \\
\hline 12 & 1 & 1 & 1 & 1 & 0 & 0 & 0 & 1 & 0 & 0 & 1 & 0 & 1 & 1 & 1 & 1 & 2 & 16 \\
\hline 13 & 1 & 1 & 1 & 1 & 0 & 0 & 0 & 2 & 0 & 0 & 1 & 0 & 1 & 1 & 1 & 1 & 1 & 17 \\
\hline 14 & 1 & 1 & 1 & 1 & 0 & 0 & 0 & 1 & 1 & 0 & 2 & 0 & 0 & 1 & 1 & 1 & 1 & $18^{\prime}$ \\
\hline 15 & 1 & 1 & 1 & 1 & 0 & 0 & 0 & 1 & 0 & 1 & 2 & 0 & 0 & 1 & 1 & 1 & 1 & 17 \\
\hline 16 & 1 & 1 & 1 & 1 & 0 & 0 & 0 & 1 & 1 & 0 & 1 & 0 & 0 & 1 & 2 & 1 & 1 & $18^{\prime}$ \\
\hline 17 & 1 & 1 & 1 & 1 & 0 & 1 & 0 & 1 & 0 & 0 & 1 & 0 & 0 & 1 & 2 & 1 & 1 & 17 \\
\hline 18 & 1 & 1 & 1 & 1 & 0 & 0 & 0 & 1 & 0 & 0 & 2 & 0 & 0 & 1 & 2 & 1 & 1 & 17 \\
\hline 19 & 1 & 1 & 1 & 1 & 0 & 0 & 0 & 1 & 0 & 0 & 1 & 0 & 0 & 1 & 2 & 1 & 2 & 16 \\
\hline 20 & 1 & 1 & 1 & 1 & 0 & 0 & 0 & 2 & 0 & 0 & 2 & 0 & 0 & 1 & 1 & 1 & 1 & 18 \\
\hline 21 & 1 & 1 & 1 & 1 & 0 & 0 & 0 & 1 & 0 & 0 & 1 & 0 & 0 & 1 & 1 & 2 & 2 & 16 \\
\hline
\end{tabular}

Table 15: $\hat{L}(s, v)$ for $v=12$ and $s=7$. Columns $1: 4,1: 5,1: 6,1: 7,2: 6,3: 6$ are omitted, as they contain only 0 entries.

\begin{tabular}{|c||c|c|c|c|c|c|c|c|c|c|c|c|c|c|c||c|}
\hline No. & $0: 1$ & $1: 2$ & $1: 3$ & $2: 3$ & $3: 4$ & $3: 6$ & $3: 8$ & $4: 5$ & $4: 6$ & $5: 6$ & $5: 8$ & $6: 7$ & $6: 8$ & $7: 8$ & $8: 9$ & $e$ \\
\hline 1 & 1 & 1 & 1 & 1 & 1 & 0 & 0 & 1 & 0 & 1 & 1 & 1 & 1 & 1 & 1 & 14 \\
\hline 2 & 1 & 1 & 1 & 1 & 1 & 0 & 1 & 1 & 0 & 1 & 0 & 1 & 1 & 1 & 1 & 15 \\
\hline 3 & 1 & 1 & 1 & 1 & 1 & 0 & 0 & 1 & 1 & 1 & 0 & 1 & 1 & 1 & 1 & 15 \\
\hline 4 & 1 & 1 & 1 & 1 & 1 & 1 & 0 & 1 & 0 & 1 & 0 & 1 & 1 & 1 & 1 & 16 \\
\hline 5 & 1 & 1 & 1 & 1 & 1 & 0 & 0 & 1 & 0 & 2 & 0 & 1 & 1 & 1 & 1 & 15 \\
\hline
\end{tabular}

Table 16: $\hat{L}(s, v)$ for $v=12$ and $s=8$. Columns containing only 0 entries are omitted.

\begin{tabular}{|c||c|c|c|c|c|c|c|c|c|c|c|c||c|}
\hline No. & $0: 1$ & $1: 2$ & $1: 3$ & $2: 3$ & $3: 4$ & $4: 5$ & $5: 6$ & $6: 7$ & $7: 8$ & $7: 9$ & $8: 9$ & $9: 10$ & $e$ \\
\hline 1 & 1 & 1 & 1 & 1 & 1 & 1 & 1 & 1 & 1 & 1 & 1 & 1 & 13 \\
\hline
\end{tabular}

Table 17: $\hat{L}(s, v)$ for $v=12$ and $s=9$. Columns containing only 0 entries are omitted. 


\begin{tabular}{|c||c|c|c|c|c|c|c|c|c|c|c|c|c|}
\hline No. & $0: 1$ & $1: 2$ & $1: 3$ & $1: 4$ & $1: 5$ & $2: 3$ & $2: 4$ & $2: 5$ & $3: 4$ & $3: 5$ & $4: 5$ & $5: 6$ & $e$ \\
\hline 1 & 1 & 1 & 1 & 0 & 1 & 1 & 0 & 1 & 1 & 2 & 1 & 3 & 27 \\
\hline 2 & 1 & 1 & 1 & 0 & 0 & 2 & 0 & 0 & 2 & 2 & 2 & 2 & 28 \\
\hline 3 & 1 & 2 & 1 & 0 & 1 & 1 & 1 & 1 & 1 & 1 & 1 & 2 & 24 \\
\hline 4 & 1 & 2 & 1 & 0 & 0 & 2 & 0 & 1 & 1 & 2 & 1 & 2 & 27 \\
\hline 5 & 1 & 2 & 1 & 0 & 0 & 1 & 1 & 1 & 1 & 1 & 2 & 2 & 25 \\
\hline
\end{tabular}

Table 18: $\hat{L}(s, v)$ for $v=13$ and $s=5$.

\begin{tabular}{|c||c|c|c|c|c|c|c|c|c|c|c|c|c|c|c|c|c||c|}
\hline No. & $0: 1$ & $1: 2$ & $1: 3$ & $1: 4$ & $1: 5$ & $1: 6$ & $2: 3$ & $2: 4$ & $2: 5$ & $2: 6$ & $3: 4$ & $3: 5$ & $3: 6$ & $4: 5$ & $4: 6$ & $5: 6$ & $6: 7$ & $e$ \\
\hline 1 & 1 & 1 & 1 & 1 & 0 & 0 & 1 & 1 & 0 & 0 & 1 & 1 & 1 & 1 & 1 & 1 & 1 & 22 \\
\hline 2 & 1 & 1 & 1 & 0 & 0 & 0 & 2 & 1 & 0 & 0 & 1 & 1 & 1 & 1 & 1 & 1 & 1 & 23 \\
\hline 3 & 1 & 1 & 1 & 1 & 0 & 0 & 1 & 1 & 0 & 0 & 1 & 0 & 1 & 2 & 1 & 1 & 1 & 23 \\
\hline 4 & 1 & 1 & 1 & 0 & 0 & 0 & 2 & 0 & 0 & 0 & 2 & 1 & 1 & 1 & 1 & 1 & 1 & 25 \\
\hline 5 & 1 & 1 & 1 & 0 & 0 & 1 & 1 & 1 & 0 & 0 & 1 & 0 & 1 & 1 & 1 & 1 & 2 & 22 \\
\hline 6 & 1 & 1 & 1 & 0 & 0 & 0 & 1 & 1 & 0 & 0 & 1 & 0 & 1 & 1 & 2 & 1 & 2 & 23 \\
\hline 7 & 1 & 1 & 1 & 0 & 0 & 0 & 1 & 0 & 0 & 0 & 2 & 0 & 1 & 1 & 2 & 1 & 2 & 24 \\
\hline 8 & 1 & 1 & 1 & 0 & 0 & 0 & 1 & 0 & 0 & 0 & 1 & 1 & 1 & 1 & 1 & 2 & 2 & 23 \\
\hline
\end{tabular}

Table 19: $\hat{L}(s, v)$ for $v=13$ and $s=6$.

\begin{tabular}{|c|c|c|c|c|c|c|c|c|c|c|c|c|c|c|c|c|c|c|}
\hline No. & $0: 1$ & $1: 2$ & $1: 3$ & $2: 3$ & $2: 4$ & $2: 5$ & $2: 7$ & $3: 4$ & $3: 5$ & $3: 7$ & $4: 5$ & $4: 6$ & $4: 7$ & $5: 6$ & $5: 7$ & $6: 7$ & $7: 8$ & $e$ \\
\hline 1 & 1 & 1 & 1 & 1 & 1 & 0 & 0 & 1 & 1 & 0 & 1 & 0 & 1 & 1 & 1 & 1 & 1 & 20 \\
\hline 2 & 1 & 1 & 1 & 1 & 0 & 0 & 0 & 1 & 1 & 1 & 1 & 1 & 0 & 1 & 1 & 1 & 1 & 20 \\
\hline 3 & 1 & 1 & 1 & 1 & 0 & 0 & 1 & 1 & 1 & 0 & 1 & 0 & 0 & 1 & 1 & 1 & 2 & 20 \\
\hline 4 & 1 & 1 & 1 & 1 & 0 & 0 & 0 & 1 & 1 & 1 & 1 & 0 & 0 & 1 & 1 & 1 & 2 & 21 \\
\hline 5 & 1 & 1 & 1 & 1 & 0 & 0 & 0 & 1 & 1 & 0 & 1 & 0 & 1 & 1 & 1 & 1 & 2 & 20 \\
\hline 6 & 1 & 1 & 1 & 1 & 0 & 0 & 0 & 1 & 0 & 1 & 1 & 0 & 1 & 1 & 1 & 1 & 2 & 20 \\
\hline 7 & 1 & 1 & 1 & 1 & 0 & 0 & 0 & 2 & 1 & 0 & 1 & 0 & 1 & 1 & 1 & 1 & 1 & 21 \\
\hline 8 & 1 & 1 & 1 & 1 & 0 & 0 & 0 & 1 & 2 & 0 & 1 & 0 & 0 & 1 & 2 & 1 & 1 & 23 \\
\hline 9 & 1 & 1 & 1 & 1 & 0 & 1 & 0 & 1 & 1 & 0 & 1 & 0 & 0 & 1 & 2 & 1 & 1 & 22 \\
\hline 10 & 1 & 1 & 1 & 1 & 0 & 0 & 0 & 1 & 1 & 0 & 2 & 0 & 0 & 1 & 2 & 1 & 1 & 22 \\
\hline 11 & 1 & 1 & 1 & 1 & 0 & 0 & 0 & 1 & 1 & 0 & 1 & 0 & 0 & 1 & 2 & 1 & 2 & 21 \\
\hline 12 & 1 & 1 & 1 & 1 & 0 & 0 & 0 & 2 & 1 & $\overline{0}$ & 2 & 0 & 0 & 1 & 1 & 1 & 1 & 22 \\
\hline 13 & 1 & 1 & 1 & 1 & 0 & 0 & 0 & 1 & 1 & 0 & 1 & 0 & 0 & 1 & 1 & 2 & 2 & 20 \\
\hline
\end{tabular}

Table 20: $\hat{L}(s, v)$ for $v=13$ and $s=7$. Columns containing only 0 entries are omitted. 


\begin{tabular}{|c||c|c|c|c|c|c|c|c|c|c|c|c|c|c|c|c||c|}
\hline No. & $0: 1$ & $1: 2$ & $1: 3$ & $2: 3$ & $3: 4$ & $3: 5$ & $3: 6$ & $3: 8$ & $4: 5$ & $4: 6$ & $5: 6$ & $5: 8$ & $6: 7$ & $6: 8$ & $7: 8$ & $8: 9$ & $e$ \\
\hline 1 & 1 & 1 & 1 & 1 & 1 & 1 & 0 & 0 & 0 & 1 & 1 & 1 & 1 & 1 & 1 & 1 & 18 \\
\hline 2 & 1 & 1 & 1 & 1 & 0 & 1 & 0 & 0 & 1 & 1 & 1 & 1 & 1 & 1 & 1 & 1 & 18 \\
\hline 3 & 1 & 1 & 1 & 1 & 0 & 1 & 1 & 0 & 0 & 1 & 1 & 1 & 1 & 1 & 1 & 1 & 18 \\
\hline 4 & 1 & 1 & 1 & 1 & 0 & 1 & 0 & 1 & 0 & 1 & 1 & 1 & 1 & 1 & 1 & 1 & 19 \\
\hline 5 & 1 & 1 & 1 & 1 & 0 & 2 & 0 & 0 & 0 & 1 & 1 & 1 & 1 & 1 & 1 & 1 & 19 \\
\hline
\end{tabular}

Table 21: $\hat{L}(s, v)$ for $v=13$ and $s=8$. Columns containing only 0 entries are omitted.

\begin{tabular}{|c||c|c|c|c|c|c|c|c|c|c|c|c|c|c||c|}
\hline No. & $0: 1$ & $1: 2$ & $1: 3$ & $2: 3$ & $3: 4$ & $3: 7$ & $4: 5$ & $5: 6$ & $5: 7$ & $6: 7$ & $7: 8$ & $7: 9$ & $8: 9$ & $9: 10$ & $e$ \\
\hline 1 & 1 & 1 & 1 & 1 & 1 & 0 & 1 & 1 & 1 & 1 & 1 & 1 & 1 & 1 & 16 \\
\hline 2 & 1 & 1 & 1 & 1 & 1 & 1 & 1 & 1 & 0 & 1 & 1 & 1 & 1 & 1 & 17 \\
\hline
\end{tabular}

Table 22: $\hat{L}(s, v)$ for $v=13$ and $s=9$. Columns containing only 0 entries are omitted. 


\section{$\mathrm{C} \quad \mathrm{Cq}^{\star} / A(5)^{3}=\mathrm{Cq}^{\star} / \approx$}

We give here an alternative reasoning that $\mathrm{Cq}^{\infty} / \approx$ consists of exactly two elements. To this end, it suffices to take into account that $\mathrm{Cq}^{\mathbf{N}} / A(5)^{3}$ has two elements (see Table 4 in Section 6 and comments therein), and to show the following result.

Proposition 23. If $\mathscr{C}$ and $\mathscr{D}$ belonging to $\mathrm{Cq}$ are combinatorially equivalent, then there is $g \in A^{3}(5)$ such that $g(\mathscr{C})=\mathscr{D}$.

We collect indispensable notions and results before delving into the proof.

Let $v$ be a vertex of a simple graph $G$. The neighbourhood $N(v)$ of $v$ is the set of all vertices adjacent to $v$; that is, $N(v)=\{x \in V(G):\{v, x\} \in E(G)\}$. We define the twin relation $\sim$ on the vertices of $G$ as follows: $u \sim v$ if and only if $N(u)=N(v)$. Clearly, the twin relation is an equivalence. The graph $G$ is point-determining if $u \sim v$ implies $u=v$, for each pair $u, v$.

Let $X$ be a non-empty finite set and $\eta: X \rightarrow V(G)$ be an 'onto' mapping. Let $G^{\eta}$ be the graph with set of vertices $V\left(G^{\eta}\right)=X$ and set of edges $E\left(G^{\eta}\right)=\{\{u, v\} \subseteq$ $X:\{\eta(u), \eta(v)\} \in E(G)\}$. The graph $G^{\eta}$ is an instant of a blow-up of $G$.

Proposition 24. Let $G$ be a point-determining graph. If $G^{\eta}$ is a blow-up of $G$ defined by an 'onto' mapping $\eta: X \rightarrow V(G)$, then for every $x \in X$, the equivalence class of $x$ with respect to the twin relation is equal to the preimage of $\eta(x)$; that is,

$$
[x]_{\sim}=\eta^{-1}(\eta(x)) .
$$

Proof. Let $y \in \eta^{-1}(\eta(x))$; equivalently, $\eta(y)=\eta(x)$. Then

$$
u \in N(x) \Longleftrightarrow\{\eta(u), \eta(x)\} \in E(G) \Longleftrightarrow\{\eta(u), \eta(y)\} \in E(G) \Longleftrightarrow u \in N(y), \quad \forall u \in X .
$$

Thus, $y \in[x]_{\sim}$ and consequently $\eta^{-1}(\eta(x)) \subseteq[x]_{\sim}$.

Suppose now that $y \sim x$. Let $w \in V(G)$, and $z$ be an element of the preimage $\eta^{-1}(w)$. The preimage is non-empty, as $\eta$ is 'onto'. We have

$$
\{w, \eta(y)\} \in E(G) \Longleftrightarrow\{\eta(z), \eta(y)\} \in E(G) \Longleftrightarrow z \in N(y) \text {. }
$$

The same argument can be repeated with $y$ replaced by $x$. Now, since $y \sim x$, we deduce $\{w, \eta(y)\} \in E(G)$ if and only if $\{w, \eta(x)\} \in E(G)$, for every $w \in V(G)$, which shows $N(\eta(y))=N(\eta(x))$. As $G$ is point-determining, we conclude $\eta(y)=\eta(x)$. Thus, $y \in$ $\eta^{-1}(\eta(x))$, and consequently $[x]_{\sim} \subseteq \eta^{-1}(\eta(x))$. 
Lemma 25. Let $X$ and $Y$ be finite sets, and $G$ be a point-determining graph. Let $\varphi: X \rightarrow$ $V(G)$ and $\psi: Y \rightarrow V(G)$ be 'onto' mappings. If $f: X \rightarrow Y$ is a bijection such that $\{\varphi(u), \varphi(v\} \in E(G) \Leftrightarrow\{\psi \circ f(u), \psi \circ f(v)\} \in E(G)$, for every $u, v \in X$, then there is a unique automorphism $h$ of $G$ for which the following diagram commutes

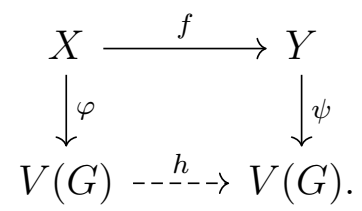

Proof. Let us first remark that our assumptions on $f$ imply that $f$ is an isomorphism of blow-up graphs $G^{\varphi}$ and $G^{\psi}$. (We shall tacitly accept that all graph-teoretic structures defined on $X$ and $Y$ relate to these graphs). In particular, we have,

$$
f(N(x))=N(f(x)), \quad \text { for every } x \in X \text {. }
$$

Let us fix an element $u \in V(G)$. Let $x, y \in \varphi^{-1}(u)$. By our proposition, we have $N(x)=$ $N(y)$, while by $(\alpha), N(f(y))=N(f(x))$. Therefore, we have proved that $f\left(\varphi^{-1}(u)\right) \subseteq$ $[f(x)]_{\sim}$. Again by our proposition there is a unique element $v \in V(G)$ such that $\psi^{-1}(v)=$ $[f(x)]_{\sim}$. Hence

$$
f\left(\varphi^{-1}(u)\right) \subseteq \psi^{-1}(v) .
$$

Since $f$ is a bijection and the family $\varphi^{-1}(u), u \in V(G)$, is a partition of $X$, its image $f\left(\varphi^{-1}(u)\right), u \in V(G)$, is a partition of $Y$. Partitions $f\left(\varphi^{-1}(u)\right), u \in V(G)$, and $\psi^{-1}(v)$, $v \in V(G)$, of $Y$ are of the same cardinality and the former is finer than the latter. Consequently, they coincide. Thus, there is a unique bijection $h: V(G) \rightarrow V(G)$ such that $f\left(\varphi^{-1}(u)\right)=\psi^{-1}(h(u))$, for every $u \in V(G)$. Clearly, $h$ is the only bijection for which our diagram commutes.

To complete the proof, it suffices to show that $h$ sends edges to edges. Let $\{u, v\}$ be an edge of $G$. Then $\varphi^{-1}(u)$ and $\varphi^{-1}(v)$ are disjoint. Let us fix $x \in \varphi^{-1}(u)$ and $y \in \varphi^{-1}(u)$. According to our definition of $h$, we have $\psi \circ f(x)=h(u)$ and $\psi \circ f(y)=h(v)$. Since $\{u, v\}=\{\phi(x), \phi(y)\}$ is an edge, it follows by our assumptions that $\{h(u), h(v)\}=$ $\{\psi \circ f(x), \psi \circ f(y)\}$ is an edge of $G$.

Proof of Proposition 23. By the combinatorial equivalence,there is an isomorphism $f: \mathscr{C} \rightarrow \mathscr{D}$. Since permutations of coordinates belong to $A^{3}(5)$, we may assume that $\varepsilon(f(I), f(J))=\varepsilon(I, J)$, whenever $I, J \in \mathscr{C}$. Equivalently, for every $i \in[3]$ and every $I, J \in \mathscr{C}, I_{i}, J_{i}$ are adjacent (have exactly one point in common) if and only if $f(I)_{i}, f(J)_{i}$ are adjacent. For each $i \in[3]$, let $\pi_{i}: \mathscr{I}^{3} \rightarrow \mathscr{I}^{1}$ be the projection $\pi_{i}(I)=I_{i}$. Observe that the graph $G$ with supp $\lambda^{\text {as }}$ its set of vertices and the adjacency inherited from $\mathscr{I}^{1}$ is point-determining. Let us set $X=\mathscr{C}, Y=\mathscr{D}, \varphi=\pi_{i}\left|\mathscr{C}, \psi=\pi_{i}\right| \mathscr{D}$. Then the sextuple $G, X, Y, f, \varphi, \psi$ satisfies the assumptions of Lemma 25. Therefore, there is an automorphism $h_{i}$ of $G$ such that the following diagram commutes 


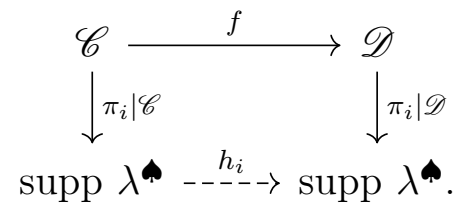

It is easy to check that $h_{i}$ extends (in a unique way) to an automorphism $g_{i} \in \operatorname{Aut}(5)$. Therefore, $h_{1} \times h_{2} \times h_{3}$ is a restriction of certain $g \in \operatorname{Aut}(5)^{3} \subset A(5)^{3}$. Moreover, observe that $\pi_{1}\left|\mathscr{C} \times \pi_{2}\right| \mathscr{C} \times \pi_{3} \mid \mathscr{C}$ is the identity mapping of $\mathscr{C}$. The identity map of $\mathscr{D}$ can be expressed in a similar way. Consequently, the following diagram commutes

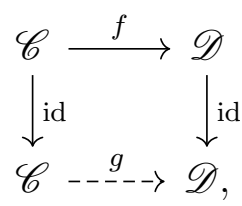

which shows that $g(\mathscr{C})=\mathscr{D}$.

\section{References}

[1] T. Achterberg, SCIP: solving constraint integer programs, Math. Prog. Comp. 1 (2009), 1-41.

[2] N. Alon, Neighborly families of boxes and bipartite coverings, in: The Mathematics of Paul Erdős II, eds. R. L. Graham, J. Nešetřil, S. Butler, Springer-Verlag, Berlin Heidelberg 1997, 27-31.

[3] F. Bagemihl, A conjecture concerning neighboring tetrahedra, Amer. Math. Monthly 63 (1956), 328-329.

[4] V. J. D. Baston, Some Properties of Polyhedra in Euclidean space, Pergamon Press, Oxford 1965.

[5] J. Ericson, S. Kim, Arbitrarily large neighborly families of congruent symmetric convex 3-polytopes, in: Discrete Geometry: In Honor of W. Kuperberg 60th Birthday, ed. A. Bezdek, Marcel Dekker Inc, New York 2003, 286-296.

[6] Z. Füredi, Matchings and Covers in Hypergraphs, Graph. Combinator. 4, 115-206.

[7] S. Furino, B. Gamble, J. Zaks, Nearly-neighbourly tetrahedra and the decomposition of some multigraphs, Res. Report. CORR 88-22 (1988), University of Waterloo, Canada.

[8] R. L. Graham and H. O. Pollak, On the addressing problem for loop switching, Bell Syst. Tech. J. 50 (1971), 2495-2519.

[9] A. P. Kisielewicz, K. Przesławski, No more than $2^{d+1}-2$ nearly neighbourly simplices in $\mathbb{R}^{d}$, submitted.

[10] T. Koch, Rapid mathematical programming, Technische Universität Berlin, 2004. 
[11] A. V. Kuzminykh, On neighborly families of convex bodies, J. Geom. 79 (2004), 134-145.

[12] A. Makhorin, GNU Linear Programming Kit, GNU Software Foundation, 2010.

[13] M. A. Perles, At most $2^{d+1}$ neighborly simplices in $E^{d}$, Annals of Discrete Math. 20 (1984), 253-254.

[14] J. D. Simon, Bounds on the cardinalities of nearly neighborly and neighborly families of polytopes, Ph. D. Thesis, University of Illinois at Urbana-Champaign, 1989.

[15] J. D. Simon, Bounds on the cardinalities of nearly neighborly families of quadrilaterals, Geometriae Dedicata 39 (1991), 173-212.

[16] J. Zaks, Bounds of neighborly families of convex polytopes, Geometriae Dedicata 8 (1979), 279-296.

[17] J. Zaks, Nearly-neighborly families of tetrahedra and the decomposition of some multigraphs, J. Combin. Theory Ser A. 48 (1988), 147-155.

[18] J. Zaks, No nine neighborly tetrahedra exist, Memoirs of the AMS 447, 1991.

[19] J. Zaks, Neighborly families of $2^{d}$ simplices in $E^{d}$, Geometriae Dedicata 11 (1981), 505-507.

[20] J. Zaks, How does a complete graph split into bipartite graphs and how are neighbourly cubes arranged?, Amer. Math. Monthly 92 (1985), 568-571.

[21] The GAP Group, GAP - Groups, Algorithms, and Programming, Version 4.7.9, (2015). https://www.gap-system.org 